\title{
NUCLEAR MOMENTS AND NUCLEAR STRUCTURE
}

Annual Progress Report

Leon Madansky and Yung Keun Lee

The Johns Hopkins University

Baltimore, Maryland

August 1, 1979 - Ju1y 31, 1980

PREPARED FOR THE DEPARTMENT OF ENERGY UNDER CONTRACT

DE-AS02-76ER03274 


\section{DISCLAIMER}

This report was prepared as an account of work sponsored by an agency of the United States Government. Neither the United States Government nor any agency Thereof, nor any of their employees, makes any warranty, express or implied, or assumes any legal liability or responsibility for the accuracy, completeness, or usefulness of any information, apparatus, product, or process disclosed, or represents that its use would not infringe privately owned rights. Reference herein to any specific commercial product, process, or service by trade name, trademark, manufacturer, or otherwise does not necessarily constitute or imply its endorsement, recommendation, or favoring by the United States Government or any agency thereof. The views and opinions of authors expressed herein do not necessarily state or reflect those of the United States Government or any agency thereof. 


\section{DISCLAIMER}

Portions of this document may be illegible in electronic image products. Images are produced from the best available original document. 


\section{NUCLEAR MOMENTS AND NUCLEAR STRUCTURE}

\section{Annual Progress Report}

Leon Madansky and Yung Keun Lee

The Johns Hopkins University

Baltimore, Maryland

August 1, 1979 - July 31, 1980

PREPARED FOR THE DEPARTMENT OF ENERGY UNDER CONTRACT

DE-AS02-76ER03274

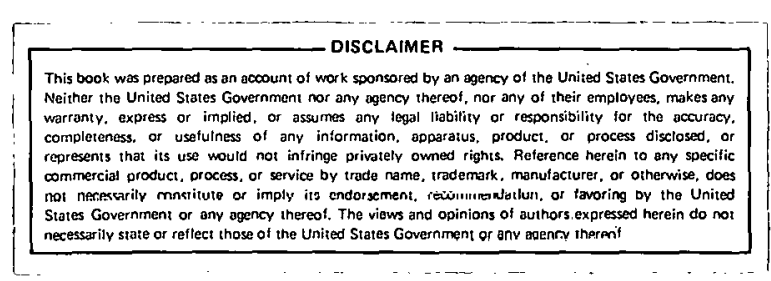




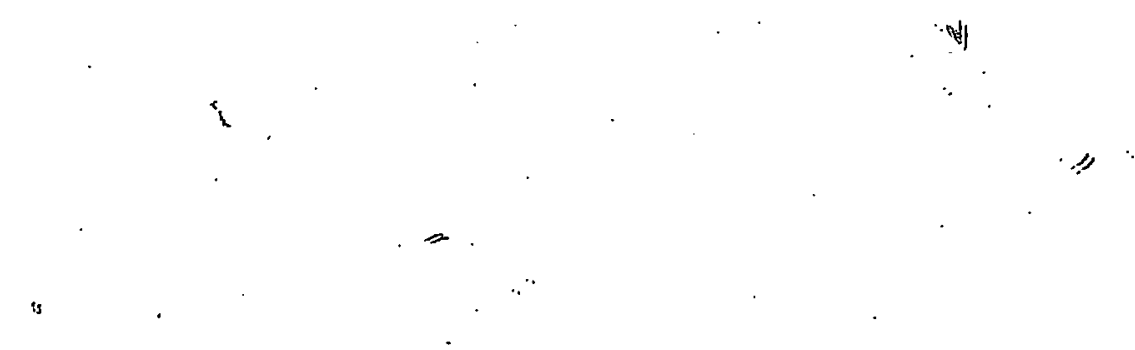

THIS PAGE

WAS INTENTIONALLY

LEFT BLANK 
RESEARCH STAFF :

$$
\begin{aligned}
& \text { Professor Leon Madansky, Co-Research Director } \\
& \text { Professor Yung Keun Lee, Co-Research Director } \\
& \text { Professor James Calvin Walker }
\end{aligned}
$$

\author{
Mr. A. J. Caffrey \\ Mr. Timothy J. Haliman \\ Mr. Ron Levin \\ Mr. Edward McIntyre, Jr. \\ Mr. Raymond Zich
}

Research Assistant

Research Assistant

Research Assistant

Research Assistant

Research Assistant 


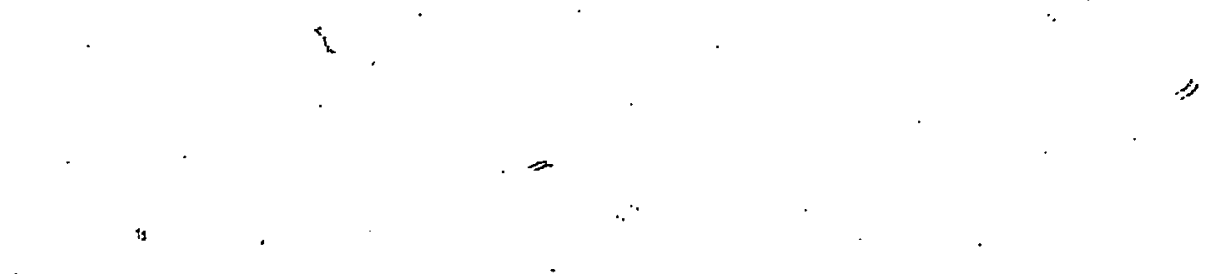

THIS PAGE

WAS INTENTIONALLY

LEFT BLANK 
I. High Energy Gamma Ray and Pion Production from Relativistic Heavy-Ion Central Collisions: Introduction

II. Limits on Threshold Phenomena in Central Collisions of $12 \mathrm{C}+\mathrm{Pb}$ from $1-2 \mathrm{GeV} / \mathrm{n}$

III. Progress in the Analysis of Meson Production in ${ }^{12} \mathrm{C}$ $+\mathrm{Pb}$ Collisions

IV. High Energy Gamma Radiation as a Probe of Nuclear Matter Produced in Heavy Ion Collisions

V. Alpha-alpha Collisions at U1trarelativistic Energies

VI. New Precision Pionic Mass Measurement and Mu-neutrino Mass Value from Pionic Atoms

VII. The Neutron and Gamma-ray Correlation in the $\pi^{-}$-capture in $165 \mathrm{Ho}$ and $181 \mathrm{Ta}$

VIII. The Neutron-neutron and the Neutron-gamma Ray Correlation in the Reaction $12 \mathrm{C}+158_{\mathrm{Gd}}$ 


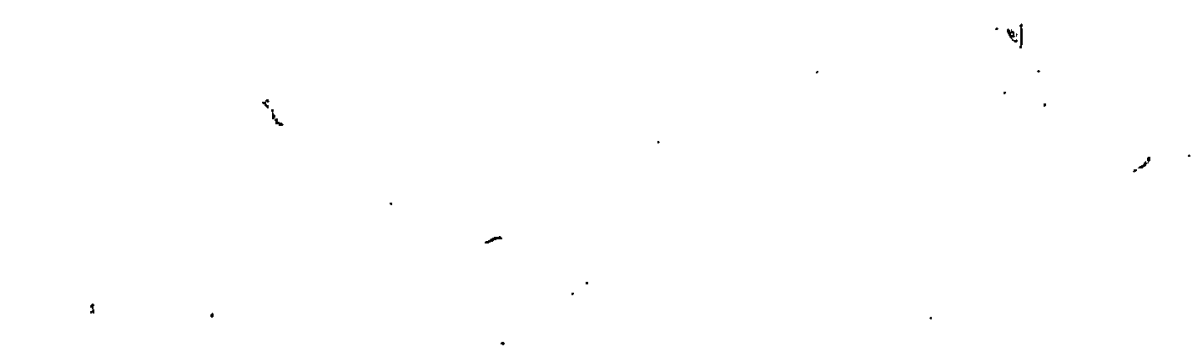

THIS PAGE

WAS INTENTIONALLY

LEFT BLANK 
INTRODUCTION

This report is a review of the activities of the period from August 1, 1979 through July 31, 1980. The results from the relativistic heavy-ion central collisions from the Bevatron are updated, and now represent the most recent evaluation of an energy scan in a search for phase transitions in nuclear matter. The effective cross-section for the pion production, and additional information including the multiplicity distributions and $\pi-\pi$ correlations are obtained.

Preparation is underway for an approved experiment using heavier projectiles at the Bevalac, searching for the unusual production of high energy gamma-rays and lepton pairs as signatures for neutral mesons. The importance of such events as an evidence of the onset of phase transition is discussed. A brief summary is given for an alphaalpha collision experiment at the CERN ISR in the search for unusual behavior in secondary hadron production.

The measurement of the pionic $X$-rays using a bent crystal spectrometer is now analyzed to yield the new value for pionic mass, and upper limit for the muonic neutrino mass. Preparation is underway for the approved experiment at the TRIUMF for the study of the neutron and gamma ray correlation in the $\pi$-capture. A parallel experiment was carried out using heavy-ion beams which atso served to test our experimental arrangement for the TRIUMF experiment. 
I. HIGH ENERGY GAMMA RAY AND PION PRODUCTION FROM RELATIVISTIC HEAVY-ION CENTRAL COLLISIONS

W. DeJarnette,* T. Hallman, E. McIntyre, Jr., J."C. Walker and L. Madansky, J. Carroll,t A. Sagle,t and R. J. Semper**

INTRODUCTION

An interim report on the status of this program was given in Progress Report c00-3274-24, covering activities through the year 1979. Part I of this report will present an updated version of the results of the experiment completed at the Bevatron. It represents the most recent evaluation of an energy scan in a search for phase transitions in nuclear matter. The data, however, can also be interpreted in terms of probabilities for production of pi-mesons in central collisions which has its own intrinsic interest. Part II, an addendum to the energy scan report, is therefore. included and presents some of these numbers. These effective cross sections are derived from extensive Monte Carlo calculations based on some of the known charged meson data and represent some of the first measurements of this kind. Additional information on multiplicity distributions for charged pions, evidence of $\pi-\pi$ correlations and some new data on the moments of energy distributions obtained from scintillators surrounding the target in central collisions will also be

* Presently with Data Acquisition Corporation, Towson, Maryland

+ From Lawrence Berkeley Laboratory, Berkeley, Cal ifornia

** Presently at the Exploratorium, San Francisco, California 
given.

The natural extension of this work to heavier projectiles (and higher energies in the future) was discussed in a contribution to a Workshop on Relativistic Heavy Ion Physics at Berkeley (February, 1980). This survey of the types of experiments involving the production of neutral mesons and the search for unusual production of high energy gamma-rays is given in Part III. In this connection an approved Hopkins experiment to be completed in the fall of 1980 was motivated in part by the expectation that, in addition to high energy gamma radiation, lepton pairs could also provide a signature for phase changes in hot hadronic matter. A portion of this run will be devoted to the study of backgrounds produced from sources other than direct electron-positron pair production. A recent paper by Domokos and Goldman has emphasized the importance of this search as a possibly clear signature of the onset of a phase transition from ordinary hadronic matter in heavy-ion central collisions. The Hopkins group work in heavy ion physics wili undoubtedly take one of these directions in the future.

Finally, a short summary, Part IV, of an experiment planned for the latter part of July, 1980, on alpha-alpha collisions at the CERN ISR $(15 \mathrm{GeV} / \mathrm{n})$ is also given. This project will be carried out as experiment R807 (Albrow, spokesman, in collaboration with a group at the University of Pennsylvania under the direction of Sherman Frankel). The motivation for this experiment is the search for unusual behavior in secondary hadron production. That is to say, any observation which shows a qualitative deviation in the physics of these collisions, different 
from the results expected from the independent collision of the four projectiles and four targets could be a signature of the aforementioned phase transitions and open up a very exciting direction for future heavy ion research. 
II. LIMITS ON THRESHOLD PHENOMENA IN CENTRAL COLLISIONS OF ${ }^{12} \mathrm{C}+\mathrm{Pb}$ FROM $1-2 \mathrm{GeV} / \mathrm{n}$

W. DeJarnette, T. Hallman, E. McIntyre, J. C. Walker, L. Madansky, J. Carroll, A. Sagle, R. Semper

\section{ABSTRACT}

Central Collisions of ${ }^{12} \mathrm{C}$ and $\mathrm{Pb}$ nuclei have been examined at six beam energies from $1-2 \mathrm{GeV} / \mathrm{n}$ for possible threshold phenomena, using neutral and charged pi meson production as a probe. The results indicate that although pion production increases by approximately a factor of two in this energy range, the increase is a smooth function of beam energy showing no such effects within statistical errors of $\cong 5 \%$ for the most central collisions.

In recent years, the possibility of learning new information about the nuclear equation of state has made central collisions of relativistic heavy ions the subject of much theoretical and experimental work. Several experimental studies $^{7-3}$ have produced important information concerning the multiplicity distributions and momentum spectra of nucleons and. pions observed in these reactions. To help explain these data, a number of models ${ }^{4-6}$ have been constructed, each of which can qualitatively predict certain aspects of the observed spectra.

One direction not yet explored is the possibility of a phase transition from hadronic matter to quark matter ${ }^{7}$ in central collisions of heavy ions. ${ }^{8}$ Chapline and Kerman have predicted that a decrease in 
pion production might be used as a signature of the onset of this phase transition. This effect is expected to be relatively small, ${ }^{9}$ and would probably go unobserved unless searched for systematically, with techniques involving high statistical accuracy. In this letter, we report the first results of such a study. Using the rate of production of high energy gamma rays from $\pi^{\circ}$ decay and the rate of production of fast charged pions $(\beta \geq .8)$ as a probe, central collisions of ${ }^{12} \mathrm{C}$ and $\mathrm{Pb}$ nuclei have been examined at six beam energies from $1-2 \mathrm{GeV} / \mathrm{n}$ for possible threshold effects.

It is generally accepted that central coilisions are characterized by high multiplicities of energetic reaction fragments distributed over the full solid angle. For this reason, an eight-element wide angle scintillator array was used as a central collision identifying trigger (Figure 1). The required coincidence of four upstream trigger paddles and four downstream ${ }^{10}$ trigger paddles yielded the trigger cross sections listed in Table $1,{ }^{11}$ which are consistent with an absolute total inelastic cross section of $(1.3 \pm 0.2)$ barns.

A deviation of the pion production cross section from smooth behavior would be significant to the extent that other parameters characterizing the collision (e.g. multiplicity, angular distributions, etc.) vary slowly and continuously as the bombarding energy changes. A useful monitor in this regard is the trigger rate. A graph of trigger cross section versus beam energy (Figure 2a) shows clearly that the factors determining event selection, such as detector acceptance and event topology, vary smoothly and monotonically as a function of beam energy. 


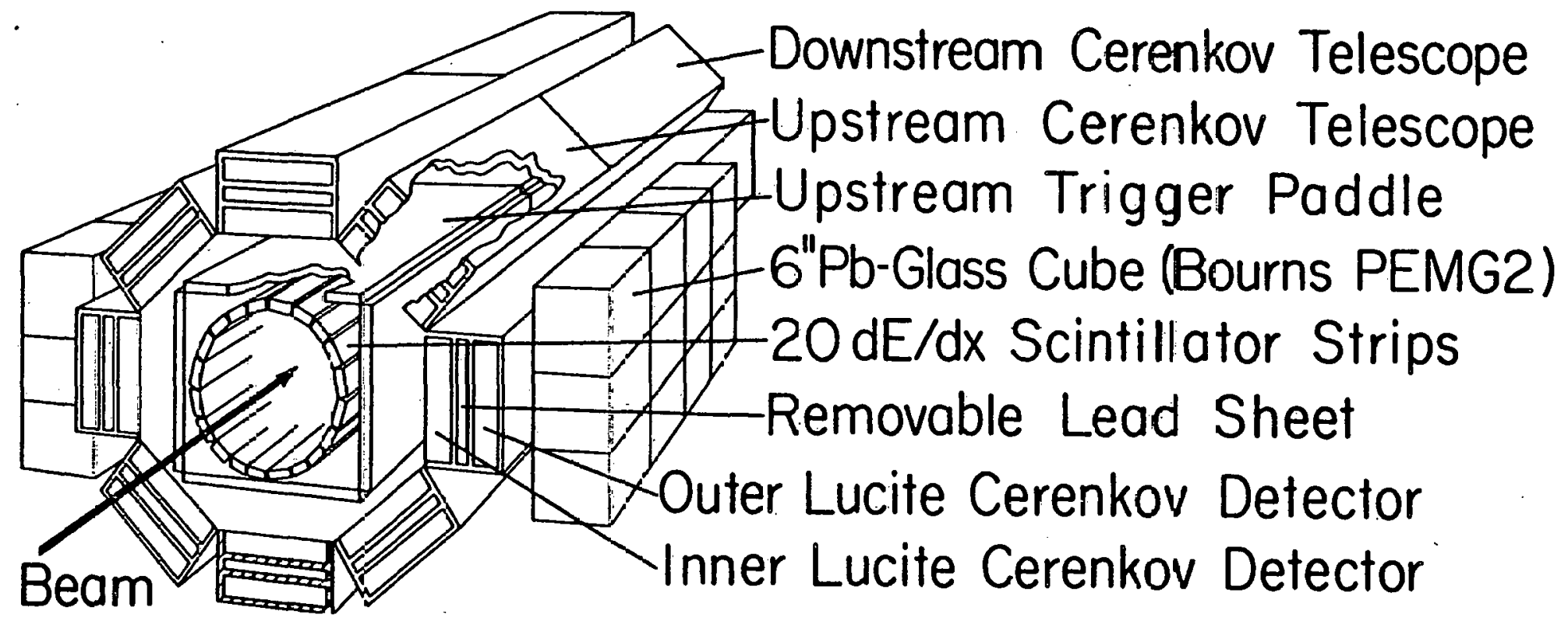

Figure 1. A front view of the entire detector system. The $d E / d x$ scintillators were located interior to the trigger counters. Lead glass walls, one on either side of the beam, were placed exterior to the Cerenkov telescope arrays. 


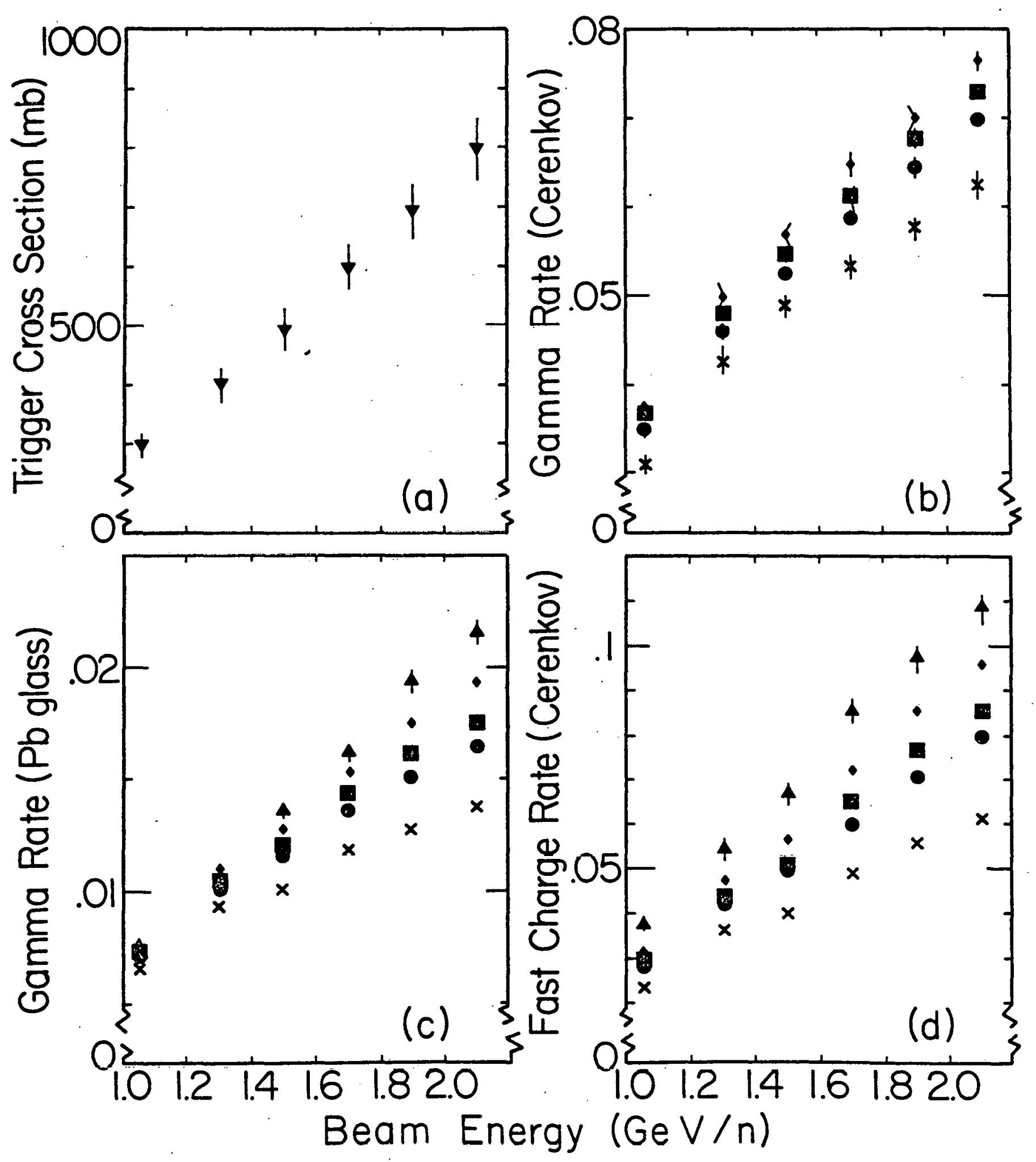

Figure 2. (a) Trigger crosssections as a function of beam energy. (b) High energy gamma production, per upstream Cerenkov telescope, per event, as a function of beam energy. (c) Gamma ray production greater than $50 \mathrm{MeV}$, per upstream $\mathrm{Pb}$ glass counter, per event, as a function of beam energy. (d) Fast charged particle production, per upstream Cerenkov telescope, per event, as a function of beam energy. cut $\emptyset, 0$ cut 1, cut $2, \mathbb{A}$ cut 3 , $\boldsymbol{X}$ cut 4 ; where not shown statistical errors are negligible. 
To determine charged particle multiplicity distributions, ${ }^{12}$ upstream and downstream $\mathrm{dE} / \mathrm{dx}$ counter systems were used (Figure 1). Each counter system was constructed of twenty 1.1 " wide NE110 scintillators, $16 \frac{1}{4} "$ long $x \frac{1}{4} "$ thick. Non-target associated background events could be rejected on the basis of a unique low multiplicity signature in the downstream $\mathrm{dE} / \mathrm{dx}$ counter system, limiting this type of background to less than $1 \%$ of the data.

Upstream and downstream Cerenkov systems were used to detect high energy gamma rays from $\pi^{\circ}$ decay and charged pions with energies greater than $150 \mathrm{MeV}$. Each detector system consisted of eight Cerenkov telescopes arrayed cylindrically about the beam (Figure 1). The Cerenkov telescopes were constructed of two $16^{\prime \prime} \times 9^{\prime \prime} \times 1 \frac{11}{4} "$ pieces of UVT lucite separated by a removable sheet of $\frac{2}{4} "$ thick lead. A high energy gamma ray would pass through the first lucite counter undetected, and convert in the lead sheet to a shower which would be detected in the second luclte counter. Monte Carlo calculations ${ }^{13}$ indicated that the effective efficiency for this process would be approximately $50 \%$. The number of high energy gamma rays per event was determined by the difference between this gamma signature rate with and without the lead converter. ${ }^{11}$ The Cerenkov telescopes were also efficient for measuring the production of charged pions with energies greater than $150 \mathrm{MeV}$, and protons with energies greater than $600 \mathrm{MeV}$. Such fast charged particles. would be detected in both pieces of UVT lucite. Taking into account known proton inclusive spectra, and the minimum energy required for a proton to be detected in the Cerenkov system; most fast charged particles 
Table 1. Trigger cross section and average $\mathrm{dE} / \mathrm{dx}$ multiplicity as a function of beam energy. filso indicated are the specific multiplicity requirements for data cuts $0-4$.

\begin{tabular}{lcccccc}
\hline Beam energy $(\mathrm{GeV} / \mathrm{n})$ & 1.05 & 1.3 & 1.5 & 1.7 & 1.9 & 2.1 \\
\hline Trigger Cross Section (mb) & $297 \pm 20$ & $399 \pm 27$ & $494 \pm 33$ & $602 \pm 40$ & $694 \pm 46$ & $801 \pm 53$
\end{tabular}

Average total multiplicity per event $\quad 18.17 \pm .02 \quad 19.99 \pm .07: 21.05 \pm .07 \quad 21.75 \pm .0722 .67 \pm .07 \quad 23.10 \pm .05$

$\begin{array}{lllllll}\text { Average upstream multiplicity per event } & 7.45 \pm .02 & 8.96 \pm .03 & 9.70 \pm .03 & 9.70 \pm .03 & 10.60 \pm .03 & 10.59 \pm .02\end{array}$

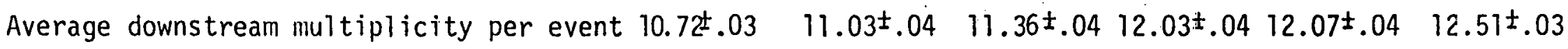

Required multiplicity for:

\begin{tabular}{|c|c|c|c|c|c|c|}
\hline cut $\emptyset$ upstream & $\geq 0$ & $\geq 0$ & $\geq 0$ & $\geq 0$ & $\geq 0$ & $\geq 0$ \\
\hline downstream & $\geq 8$ & $\geq 8$ & $\geq 8$ & $\geq 3$ & $\geq 8$ & $\geq 8$ \\
\hline cut 1 upstream & $\geq 5$ & $\geq 6$ & $\geq 6$ & $\geq 7$ & $\geq 8$ & $\geq 8$ \\
\hline downstream & $\geq 8$ & $\geq 9$ & $\geq 10$ & $\geq 10$ & $\geq 10$ & $\geq 10$ \\
\hline cut 2 upstrean & $\geq 7$ & $\geq 9$ & $\geq 9$ & $\geq 10$ & $\geq 11$ & $\geq 11$ \\
\hline downstream & $\geq 10$ & $\geq 11$ & $\geq 12$ & $\geq 12$ & $\geq 12$ & $\geq 13$ \\
\hline cut 3 upstream & $\geq 10$ & $\geq 12$ & $\geq 12$ & $\geq 13$ & $\geq 14$ & $\geq 14$ \\
\hline downstream & $\geq 13$ & $\geq 13$ & $\geq 14$ & $\geq 14$ & $\geq 14$. & $\geq 15$ \\
\hline cut 4 upstream & $<7$ & $<9$ & $<9$ & $<10$ & $<11$ & $<.11$ \\
\hline downs tream & $<10, \geq 8$ & $<\quad 17, \geq 8$ & $<12, \geq 8$ & $<12, \geq 8$ & $<12, \geq 8$ & $<13, \geq 8$ \\
\hline
\end{tabular}


observed in this system would be charged pions.

Two walls of lead glass, one on either side of the beam, were also used to detect high energy gamma rays at large polar angles (Fig. 1). Each wall was subdivided into four adjacent columns, each column consisting of 3 six-inch cubes of Bourns PEMG2 Pb-glass. Each cube was adjusted to measure energies up to approximately $250 \mathrm{MeV}$, with a resolution of $30-50 \%$. Eighty-one percent of each lead glass wa 11 was shielded by an upstream Cerenkov telescope, which eliminated spurious counts due to fast charged particles.

To conduct this energy search, ${ }^{12} \mathrm{C}$ beams at six different energies, $1.05,1.3,1.5,1.7,1.9$, and $2.1 \mathrm{GeV} / \mathrm{n}$, were used. Beam intensities typically of the order of $5 \times 10^{5}$ particles per pulse, were monitored using an independent ionization chamber. ${ }^{15}$ The target was a foil of natural lead $\left(430 \mathrm{mg} / \mathrm{cm}^{2}\right)$, placed at a position selected to maximize the resultant central collision trigger rate at $1.05 \mathrm{GeV} / \mathrm{n}$. The target position was kept fixed throughout the experiment. Approximately $2 \times 10^{5}$ events were collected at each of the six ${ }^{12} c$ beam energies.

To date, double differential cross sections for $\pi^{ \pm}$production have been measured for only two of the energies considered in this experiment, and no such data exists for $\pi^{u}$ production. Therefore, although Monte Carlo estimates can be made, the exact dependence of these spectra upon energy is largely unknown, precluding a precise determination of absolute pion production cross section at each individual beam energy. Consequently, to emphasize the relative behavior of the pion production as a function of energy, and to avoid unnecessarily 
introducing errors which are not common to all energy runs, the experimental results, shown in Figs. $2 b, c, d$, have been presented in the form of count rate per event per detector system as a function of beam energy. ${ }^{16}$ To investigate the role which impact parameter may play. in these processes the results have also been presented as a function of multiplicity. ${ }^{17}$. The cut 0 requirement eliminates non-target related background events. This cut is common to all beam energies and comprises the most general class of events. The specific multiplicity ranges associated with cuts 1-4 (Table 1) select events from comparable regions of the multiplicity distribution at each beam energy (e.g. the cut 2 multiplicity requirement selects events of greater than average multiplicity in each case). Cuts 1, 2, and 3 are characterized by successively higher multiplicity requirements, while cut 4 is a low multiplicity requirement. The errors shown, which are due to counting statistics, are generalily smaller than $\sim 1 \%$ for events satisfying the cut 0 requirement, and generally smaller than $\sim 5 \%$ for the most central collisions which satisfy the cut 3 requirement.

Fig. $2 b$ shows the rate of high energy gamma production measured by the upstream Cerenkov system. Similarly, the rate of high energy gamma production in the upstream $\mathrm{Pb}$-glass column is shown in Fig. 2c. In this system, a minimum energy requirement of $50 \mathrm{MeV}$ per $\mathrm{Pb}-\mathrm{glass}$ cube eliminated low energy gamma rays. Figure $2 \mathrm{~d}$ shows the rate of production of fast charged particles in the upstream Cerenkov telescopes. It is interesting to note that there are generally more 
high energy gamma rays and fast charged pions produced in high multiplicity events than in low multiplicity events. From the data of cuts $0-3$ it is generally evident that, within statistics, the production of charged and neutral $\pi$ mesons smoothly increases with bombarding energy from $1-2 \mathrm{GeV} / \mathrm{n}$.

In conclusion, although pion production increases by approximately a factor of two ${ }^{18}$ in the energy range studied, the increase is a smooth function of beam energy showing no evidence of threshold phenomena within statistical errors of $\sim 5 \%$ for the most central collisions.

We are indebted to the staff of the Lawrence Berkeley Laboratory, and especially to E. Whipple, for their assistance in running this experiment.

\section{REFERENCES :}

1. See review of J. R. Nix, Los Alamos Report 77-2952, e.g., J. Gosset, H. H. Gutbrod, W. G. Meyer, A. M. Yoskanzer, A. Sandová1, R. Stock, G. D. Westfall, Phys. Rev. C16, 629 (1977).

2. J. Chiba, K. Nakai, I. Tanihata, S. Nagamfya, H. Bowman, J. Ingersoll, and J. 0. Rasmussen, Phys. Rev. C20, 1336 (1979).

3. S. Y. Fung, W. Gorn, G. P. Kernan, F. F. Liu, J. J. Lu, Y. T. Oh, L. Schroeder, H. Steiner, Phys. Rev. Lett. 40, 292 (1977).

4. J. Gosset, J. I. Kapusta, and G. D. Westfall, Phys. Rev. C18, 844 (1978); G. D. Westfa11, J. Gosset, P. J. Johansen, A. M. Poskanzer, W. G. Meyer, H. H. Gutbrod, A. Sandoval, and R. Stock, Phys. Rev. Lett. 37,1202 (1976). 
5. A. A. Amsden, J. N. Ginocchio, F. H. Harlow, J. R. Nix, M. Danos, E. C. Halbert, R. K. Smith, Jr., Phys. Rev. Lett. 38, 1055 (1977); A. A. Amsden, F. H. Harlow, J. R. Nix, Phys. Rev. C15, 2059 (1977).

6. J. P. Bondorf, H. T. Feldmeier, S. I. A. Garpman, E. C. Halbert, Phys. Lett. 65B, 217 (1976); J. P. Bondorf, P. J. Siemens, S. I. A. Garpman, E. C. Halbert, Z. Phys. A279, 385 (1976); K. K. Gudima, H. Iwe, and V. D. Toneev, J. Phys. G: Nuc. Phys. $\underline{5}, 229$ (1979).

7. N. Itoh, Prog. Theor. Phys. 44, 291 (1970); F. Iachel10, W. D. Langer and A. Lande, Nuc. Phys. A219, 612 (1974); J. C. Collins and M. J. Perry, Phys. Rev. Lett. 34, 1353 (1975); M. Kislinger and P. Morley, Phys. Lett. 67B, 371 (1977).

8. S. A. Chin, Phys. Lett. $\underline{78 B}, 552$ (1978); K. A. 01ive, Phys. Lett. 89B, 299 (1979).

9. G. Chapline and A. Kerman, Lawrence Livermore Lab Report UCRL 30737 (1978). Chapline and Kerman estimate that a phase transition from nuclear to quark matter might be characterized by a threshold decrease in pion production of approximately $20 \%$.

10. Throughout this experiment, the convention was adopted that the beam flowed from 'upstream' to 'downstream'. A given counter could therefore be located upstream or downstream of the target.

11 . Errors quoted in Table 1 represent the uncertainty in the measurement of target thickness and absolute calibration of the beam monitor. These systematic errors are common to all energy runs. Statistical errors are insignificant on this scale.

12. Throughout this paper, 'multiplicity' will refer to the number of 
$\mathrm{dE} / \mathrm{dx}$ scintillators which detect a particle in a given event. Due to the high number of $\mathrm{dE} / \mathrm{dx}$ scintillators used (40), this 'multiplicity' is, to first order directly proportional to the true charged particle multiplicity. Using a measured distribution of such 'multiplicities', and an appropriate statistical analysis to allow for the possibility of several particles passing through a single scintillator, one can arrive at the most probable number of charged particles for a given reaction.

13. H. Messel and D. R. Crawford, Electron-Photon Shower Distribution Function (Pergamon Press, Oxford, 1970); P. Darriulat, E. Gygi, M. Holder, K. T. McDonald, H. G. Pugh, F. Schneider, K. Tittel, Nucl. Inst. Meth. 129, 105 (1975).

14. Low energy gamma rays constituted a background to measurements made with the Cerenkov telescopes since they produced Compton electrons which simulated the high energy gamma ray signature. Consequently, 3" 3 " NaI detectors were placed exterior to both the upstream and downstream Cerenkov telescope arrays to measure the associated spectrum of low energy gamma rays.

15. The ionization chamber was calibrated to register 1 count per $2 \times 10^{6}$ protons at $1.05 \mathrm{GeV} / \mathrm{n}$. The integrated beam current in the ionization chamber was assumed to scale with the atomic number of the projectile, velocity remaining constant.

16. For orientation, Monte Carlo estimates using the angular distributions of Ref. 2 indicate that at $1.05 \mathrm{GeV} / \mathrm{n}$ the rate of high energy gamma rays detected in the lucite Cerenkov system translates into $\sim 1 \pi^{\circ}$ 
per event (cut 0). Such estimates are not essential to the present discussion. A full description will be given in a future publication.

17. There is evidence that multiplicity and impact parameter are inversely related. R. Stock, H. H. Gutbrod, W. G. Meyer, A. M. Poskanzer, A. Sandoval, J. Gosset, C. H. King, G. King, Ch. Lukner, Nguyen Van Sen, G. D. Westfal1, K. L. Wolf, Phys. Rev. Lett. $\underline{44}$, 1243 (1980). Therefore, for a given cut, a changing multiplicity requirement corresponds to requiring a fixed range of impact parameter at all energies.

18. A stream chamber experiment studying $\pi^{-}$production with Ar projectiles incident on $\mathrm{KCL}$ indicates similar results. A. Sandoval et al., (Darmstadt, LBL, ANL collaboration, private communication). 
III. PROGRESS IN THE ANALYSIS OF MESON PRODUCTION IN ${ }^{12} \mathrm{C}+\mathrm{Pb}$ COLLISIONS T. Hallman, E. McIntyre and L. Madansky

The following notes concern some preliminary analysis of the data on the production of mesons in central collisions that have been described in the previous paper. They indicate the progress in obtaining probabilities of the numbers of mesons produced in central collisions and some additional properties that can be obtained from the $\mathrm{dE} / \mathrm{dx}$ system. These analyses should be completed in the summer of 1980 and will be presented in dissertations of T. Hallman and E. McIntyre.

1. Prel iminary Monte Carlo Estimates of Total Pion Production per Event

Pions produced in relativistic heavy ion central collisions are found to be distributed in momentum and angle. The distribution determines the number of pions per collision observed in each element of solid angle, and consequently the data recorded by each detector system.

In order to interpret the data in terms of number of pions per event, it is necessary to evaluate the effect of the distribution. This can be done by considering the empirical data to be a product of the number of pions per event multiplied by the "acceptance" of the detector system. The acceptance accounts for the distribution of pions, and the detection efficiency of each system. If the distribution is known, then the acceptance can be calculated by Monte Carlo technique, and the total production of pions ( in 4 pi steradians) can be independently estimated from the data in each detector system. 
The most appropriate distributions to use in the calculation of the acceptance of each system are those due to ${ }^{12} \mathrm{C}+{ }^{208} \mathrm{~Pb} \rightarrow \pi^{+-}+\mathrm{X}$ at 1.05 and $2.1 \mathrm{GeV} / \mathrm{n}$. Since these distributions are not known, reasonable experimental estimates of the charged pion distributions from inclusive data on similar reactions are assumed to be applicable, and neutral pion production is taken to be similar to charged pion production. The exact distribution assumptions will be discussed later.

If the assumed distribution is similar to the real pion distribution, and the data is corrected to represent the quantity of interest (i.e., pions/event), then all detector systems ( $\mathrm{NaI}, \mathrm{Pb}-\mathrm{glass}$, Cerenkov) should predict the same value for total pion production in the full solid angle. The consistency of the predictions from the various systems is therefore a good check on the validity of the assumed distribution.

In the calculation of the acceptance, the efficiency for each system can be modelled with respect to solid angle, and such factors as the gamma conversion efficiency of the $\frac{13}{4} " \mathrm{~Pb}$ sheets (in the Cerenkov system), the energy resolution of both $\mathrm{NaI}$ and $\mathrm{Pb}-\mathrm{glass}$, (so that a minimum energy requirement can be made) and the sharing of gamma ray showers between $\mathrm{Pb}-\mathrm{glass}$ blocks. The pion distribution can be simulated from the known inclusive data. The acceptance is then the probability, for each detector system, that a pion produced according to a given distribution and subject to the relevant efficiencies, will actually be recorded. The ratio of the data to this acceptance is the estimate of pion production per event. The acceptances are calculated from specific assumptions, and systematic errors are introduced by the approximate nature of the 
Figure 1. Compares the original and simulated cross sections for the reaction ${ }^{12} \mathrm{C}+\mathrm{Pb} \rightarrow \pi^{-}+X$ at $.8 \mathrm{GeV} / \mathrm{n}$ at various laboratory angles. 


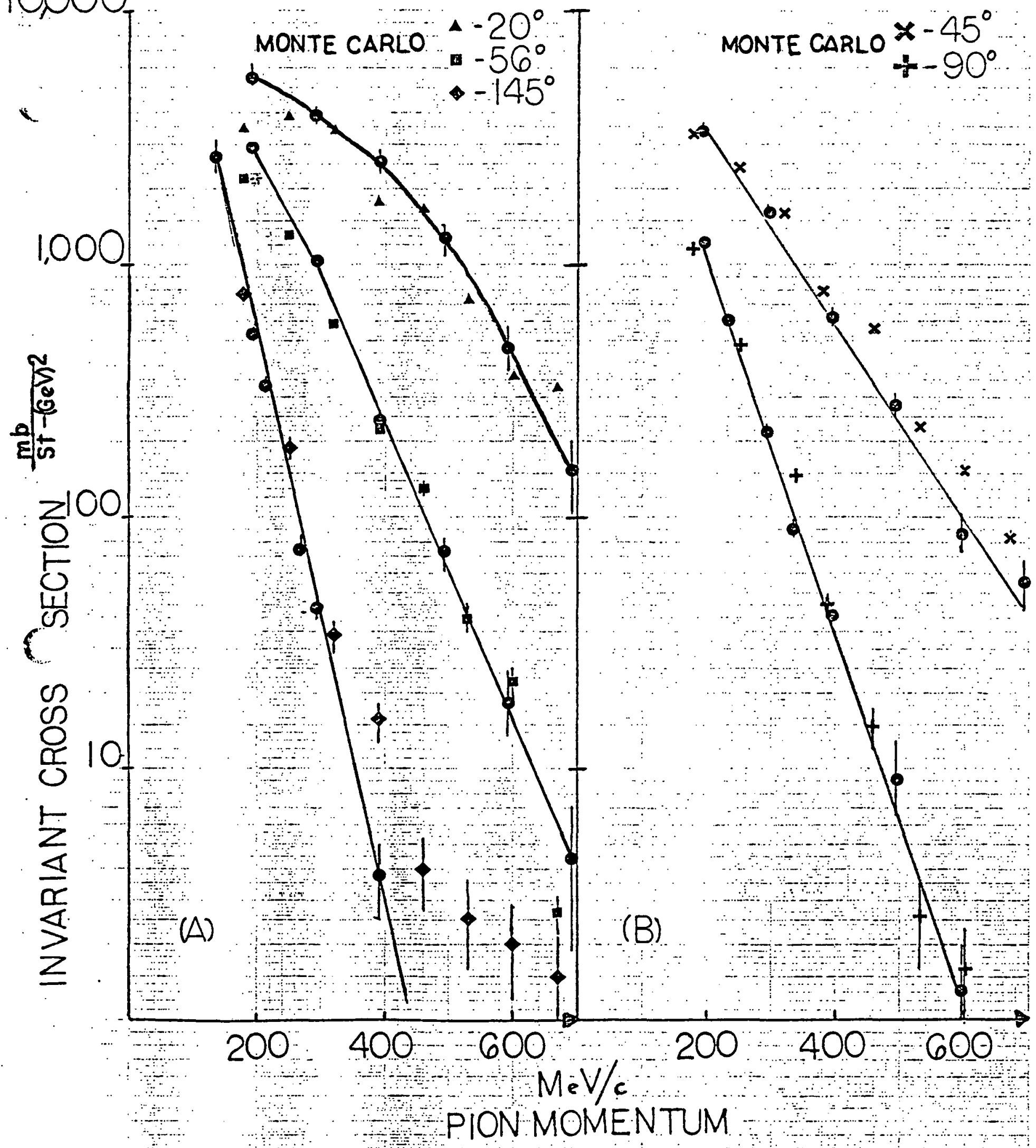

( A COMPARISON OF MONTE CARLO AND -20 EXPERIMENTAL DATA For $C+P b \stackrel{=}{\Longrightarrow} \pi+X \quad 8 \mathrm{eV} / n$ 
simulation and detector models.

Applicable experimental measurements of the pion distribution are available for the high and low beam energy portions of the data. Invariant cross sections for the inclusive reaction ${ }^{12} \mathrm{C}+{ }^{208} \mathrm{~Pb} \rightarrow \pi^{-}+X$ at a beam energy of $.8 \mathrm{GeV} / \mathrm{n}$ have been measured by Nagamiya. ${ }^{1}$. Since the pion distribution should be a slowly varying function of beam energy, these cross sections can be used to compute a distribution applicable to the $1.05 \mathrm{GeV} / \mathrm{n}$ data. The original cross section values and those computed from the Monte Carlo simulation are compared in Figure la,b. The original cross sections contain an absolute normalization.

At the high beam energy, the most appropriate experimental distribution is that of Fung, ${ }^{2}$ for ${ }^{12} \mathrm{C}+\mathrm{PbO}_{3} \rightarrow \pi^{-}+\mathrm{X}$ at $2.1 \mathrm{GeV} / \mathrm{n}$. The original and simulated distributions are compared in histograms of $\cos \left(\theta_{1 a b}\right)$ and momentum (Figure 2).

These two distributions are compared in Table 1. The Forward Throw (defined to be the ratio of the number of pions with $\cos \left(\theta_{7 a b}\right)>.5$ to that of pions with $\left.\cos \left({ }^{\theta}{ }_{1 a b}\right)<.5\right)$ charactarizes the extent to which pions are produced isotropically in $\cos \left(\theta_{1 a b}\right)$. A completely isotropic distribution would have a forward throw of .25. The Low Momentum Fraction is a measure of the behavior of the distribution for particles of low momentum. This quantity is the ratio of the number of particles with momentum less than $50 \mathrm{MeV} / \mathrm{c}$ to that of particles with momentium greater than $120 \mathrm{MeV} / \mathrm{c}$, which corresponds to the lucite Cerenkov threshold. This number is of interest because the detector systems are efficient for gammas from low momentum neutral pions, but not for low momentum charged 
pions, and the extrapolation of the distribution to low momenta must be reasonable. The major difference between distributions is the number of pions created in the forward direction. The high energy distribution has a higher fraction of pions in the forward angular region.

An .independent estimate of pion production can be made from the data in each system, and the spread of values attributed to background in the data and inaccuracies in the distribution assumptions. The results are summarized in Table 2 .

The four independent estimates of neutral pion production range from $1: 4$ /event to 2.2 /event at $1.05 \mathrm{GeV} / \mathrm{n}$ and from 3.3 /event to 4.3 /event at $2.1 \mathrm{GeV} / \mathrm{n}$. This indicates a systematic error of approximately 1 pion/event. The energy resolutions of the $\mathrm{NaI}$ and $\mathrm{Pb}-\mathrm{glass}$ systems allow more stringent restrictions on the gamma ray energy, and are therefore less subject to background. These more reliable data tend to estimate lower $\pi^{\circ}$ production. The data indicate that the ratio of charged to neutral pions is between 2 and 3 , and that the production of all types of pions increases by about a factor of 2 in this energy range.

The charged pion data is uncorrected for $\pi^{\circ}$. gammas which convert in the target or surrounding material and produce an electron of sufficient energy to create a pion signature in the Cerenkov system. A preliminary estimate of the size of this and other effects on the charged data is approximately 10\%. A more accurate estimate of the low energy gamma background will be made in the final analysis. Therefore, these estimates should be regarded as upper limits, since the entire 
Figure 2. Compares the original and simulated cosine and momentum distributions for the reaction

$$
{ }^{12} \mathrm{C}+\mathrm{PbO}_{3} \rightarrow \pi^{-}+\mathrm{X} \text { at } 2.1 \mathrm{GeV} / \mathrm{n} \text {. }
$$


100

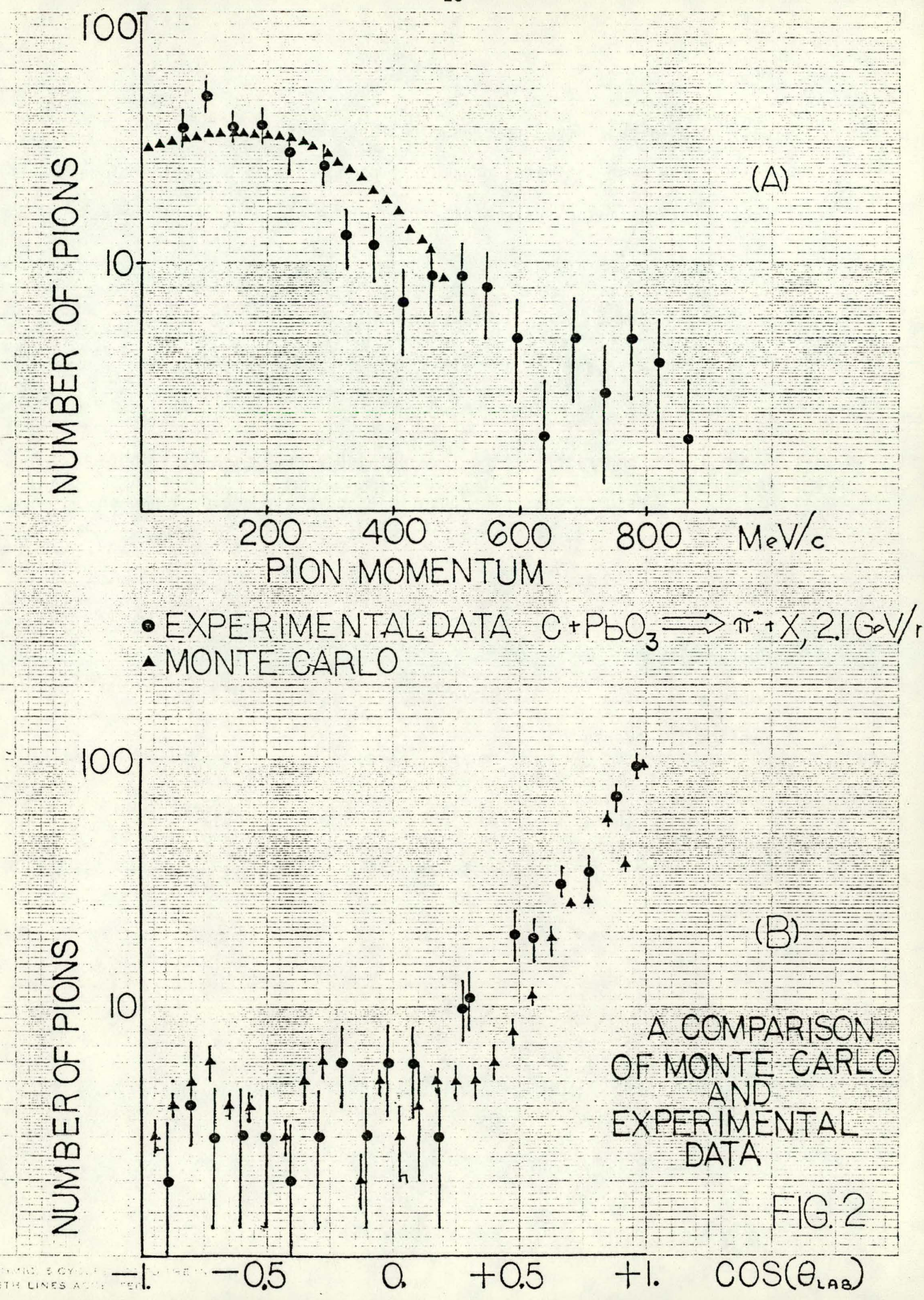


data rate is considered to be due to fast pions.

The best estimates of neutral pion production are therefore (note that estimated systematic errors are enclosed in parentheses):

\begin{tabular}{|l||l|l|}
\hline Beam Eng. & $1.05 \mathrm{GeV} / \mathrm{n}$ & $2.1 \mathrm{GeV} / \mathrm{n}$ \\
\hline$\pi^{\circ} /$ event & $1.4( \pm .5)$ & $3.6( \pm 1)$ \\
\hline
\end{tabular}

Table 1. Comparison of the low and high energy pion distribution assumptions. The quantities compared are outlined in the text.

\begin{tabular}{|c|c|c|}
\hline & $1.05 \mathrm{GeV} / \mathrm{n}$ & $2.1 \mathrm{GeV} / \mathrm{n}$ \\
\hline Forward Throw & .916 & 2.75 \\
\hline Average Momentum & $211 \mathrm{MeV} / \mathrm{c}$ & $228 \mathrm{MeV} / \mathrm{c}$ \\
\hline $\begin{array}{c}\text { Average Transverse } \\
\text { Momentum }\end{array}$ & $132 \mathrm{MeV} / \mathrm{c}$ & $137 \mathrm{MeV} / \mathrm{c}$ \\
\hline $\begin{aligned} \text { Low Momentum } & \begin{aligned} \text { Fraction } \\
\text { (LMF) }\end{aligned}\end{aligned}$ & .135 & .17 \\
\hline
\end{tabular}


Table 2. Table 2 compares estimates of total pion production in $4 \mathrm{pi}$ based on data from the various detector systems. The errors shown are statistical.

\begin{tabular}{|c|c|c|}
\hline - & \multicolumn{2}{|c|}{ Beam Energy } \\
\hline Detector & $\begin{array}{l}1.05 \mathrm{GeV} / \mathrm{n} \\
\left(\pi^{\circ} / \mathrm{gevent}\right) \\
\end{array}$ & $\begin{array}{l}2.1 \mathrm{GeV} / \mathrm{n} \\
\left(\pi^{\circ} / \text { event }\right)\end{array}$ \\
\hline $\mathrm{NaI}$ & $1.9 \pm .13$ & $4.3^{ \pm} .15$ \\
\hline Upstream Cerenkov & $2.0 \pm .05$ & $4.4 \pm .08$ \\
\hline Downstream Cerenkov & $2.2^{ \pm} .05$ & $3.3^{ \pm} .06$ \\
\hline Upstream Pb-glass & $1.4 \pm .12$ & $3.6 \pm .56$ \\
\hline & ( $\pi \pm$ /event $)$ & ( $\pi \pm$ /event) \\
\hline Upstream Cerenkov & $4.6 \pm .13$ & $9.6 \pm .2$ \\
\hline
\end{tabular}

\section{Energy Spectra}

Two signatures frequently discussed in relation to the observation of possible phase transitions in nuclear matter are 1) threshold changes in the rate of meson production and 2) discontinuous changes in the temperature of the collision which would be apparent in the energy spectra of the reaction products. We have earlier reported ${ }^{3}$ that within statistical errors of $\cong 5 \%$ for the most central collisions no threshold changes in the rate of $\pi^{ \pm}, \pi^{\circ}$ production are observed for collisions of ${ }^{12} \mathrm{C}$ ions incident on $\mathrm{Pb}$ nuclei at beam energies from $1-2 \mathrm{GeV} / \mathrm{n}$. We are currently investigating the relationship between reaction temperature and bombarding energy using the measured energy spectra of protons 
and charged pi mesons.

The $d E / d x$ counter system used in this study has been previously described in Progress Report C00-3274-24. It consisted of upstream and downstream $\mathrm{dE} / \mathrm{dx}$ counter arrays, each counter array being constructed of 20 individually adjustable phototube-scintillator assemblies mounted on a $1 / 16$ " thick aluminum cylinder approximately $9 "$ in diameter. Each phototube-scintillator assembly consisted of a $16 \frac{12}{4}$ long $\times 1$ " wide $\times \frac{3}{4} "$ thick strip of NEI10 scintillator (attenuation lenqth $\cong 4$ meters) viewed by a 12-stage, 3/4" face RCA 4802 phototube. The FWHM resolution was typically $\cong 40 \%$ for minimum ionizing cosmic rays for each scintillator detector, consistent with the resolution obtainable with thin scintillation counters. ${ }^{4}$ This resolution is approximately constant throughout the practical range of pion energies measured $(\beta \geq .8)$ but becomes considerably improved over most of the energy range appropriate to the study of proton production.

Despite the somewhat limited energy resolution for pions, the slope of the pion energy spectrum can be accurately determined and investigated as a function of beam energy. A typical pion energy spectrum is shown in Figure 3 for ${ }^{12} \mathrm{C}+\mathrm{Pb}$ at $1.05 \mathrm{GeV} / \mathrm{n}$. The fitted value of the experimental temperature at $1.05 \mathrm{GeV} / \mathrm{n}$ is $59 \mathrm{MeV}$, which compares favorably with values reported elsewhere. 5 This study will be complcted shortly for six beam energies from 1.05 to $2.1 \mathrm{GeV} / \mathrm{n}$, and the results presented in a future publication. Additionally, the slope of the pionic energy spectrum will be investigated as a function of multiplicity at each beam energy. 


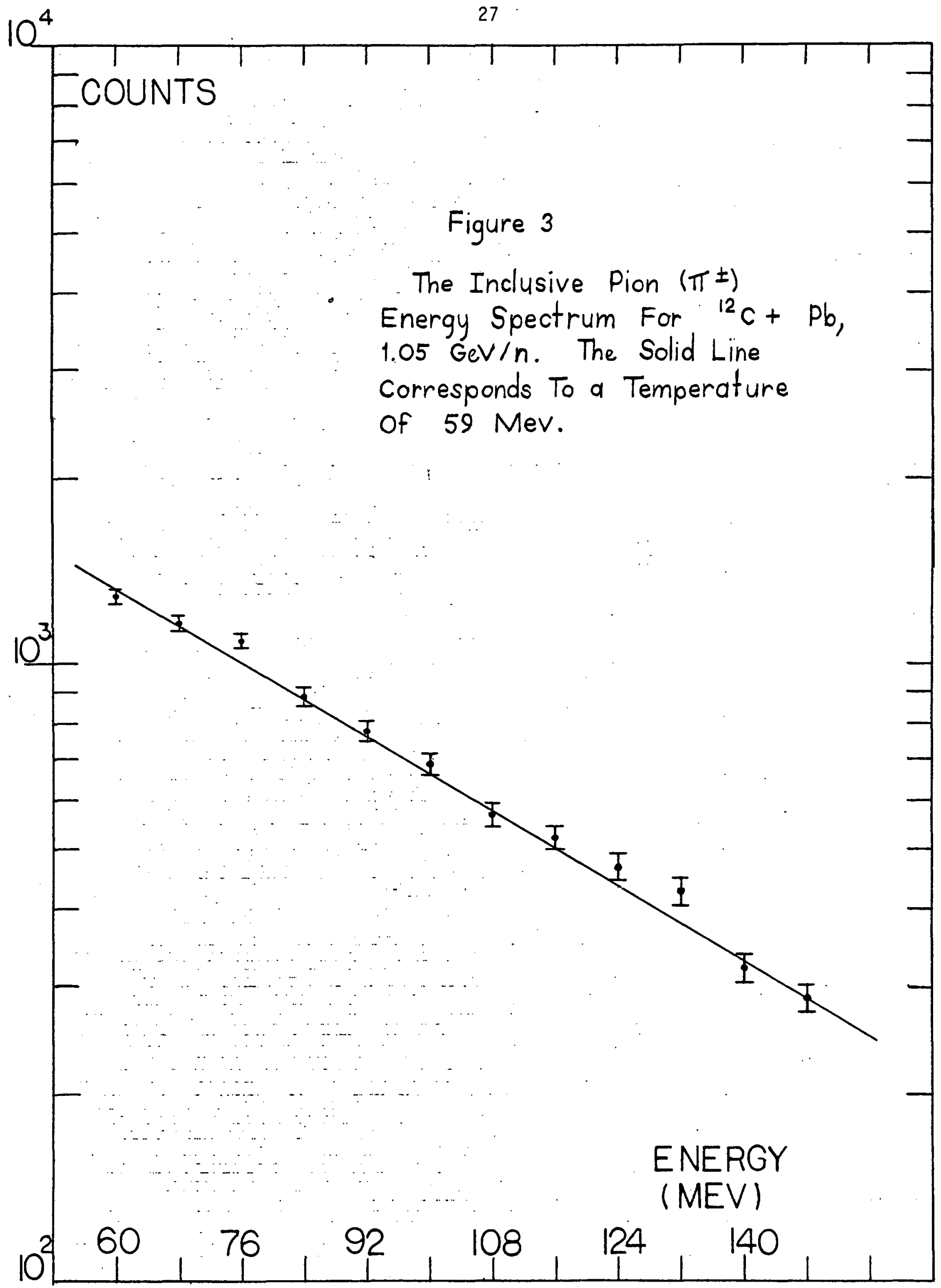


An experimental temperature can also be determined from the measured proton spectra. However, in this case the relationship between the measured temperature and the actual temperature is necessarily complicated by the changing character of the energy-loss statistics over the measured proton energy range, and by the presence of backgrounds not important in the study of pion production. ${ }^{6}$ of greater interest here, therefore, is a determination, per central collision, of the first and second moments of the exclusive proton energy distribution at each beam energy. This information has been unavailable in the proton inclusive studies made so far and consequently is of particular interest. These moment distributions should change smoothly with beam energy and can therefore be used to search for threshold phenomena which might result from a phase transition. Typical distributions of the first and second moments for exclusive, per event proton production at $1.05 \mathrm{GeV} / \mathrm{n}$ are shown in Figures 4 and 5 , respectively. The complete results of this study will be presented in the doctoral dissertations of T. J. Hallman and E. K. McIntyre.

\section{Angular Correlations}

The $\mathrm{dE} / \mathrm{dx}$ counter system also provided information concerning azimuthal angle correlations between fast charged secondaries. As shown in Figure 6 , it was observed that the probability that two fast $(\beta \geq .8)$ charges will be separated by an azimuthal angle of $\phi \pm \Delta \phi$ is peaked for small angles $\phi$. This effect has been previously observed ${ }^{7}$ for like pions produced in central collisions of heavy ions and can be interpreted 
COUNTS $(X 2.4 \mathrm{~K})$

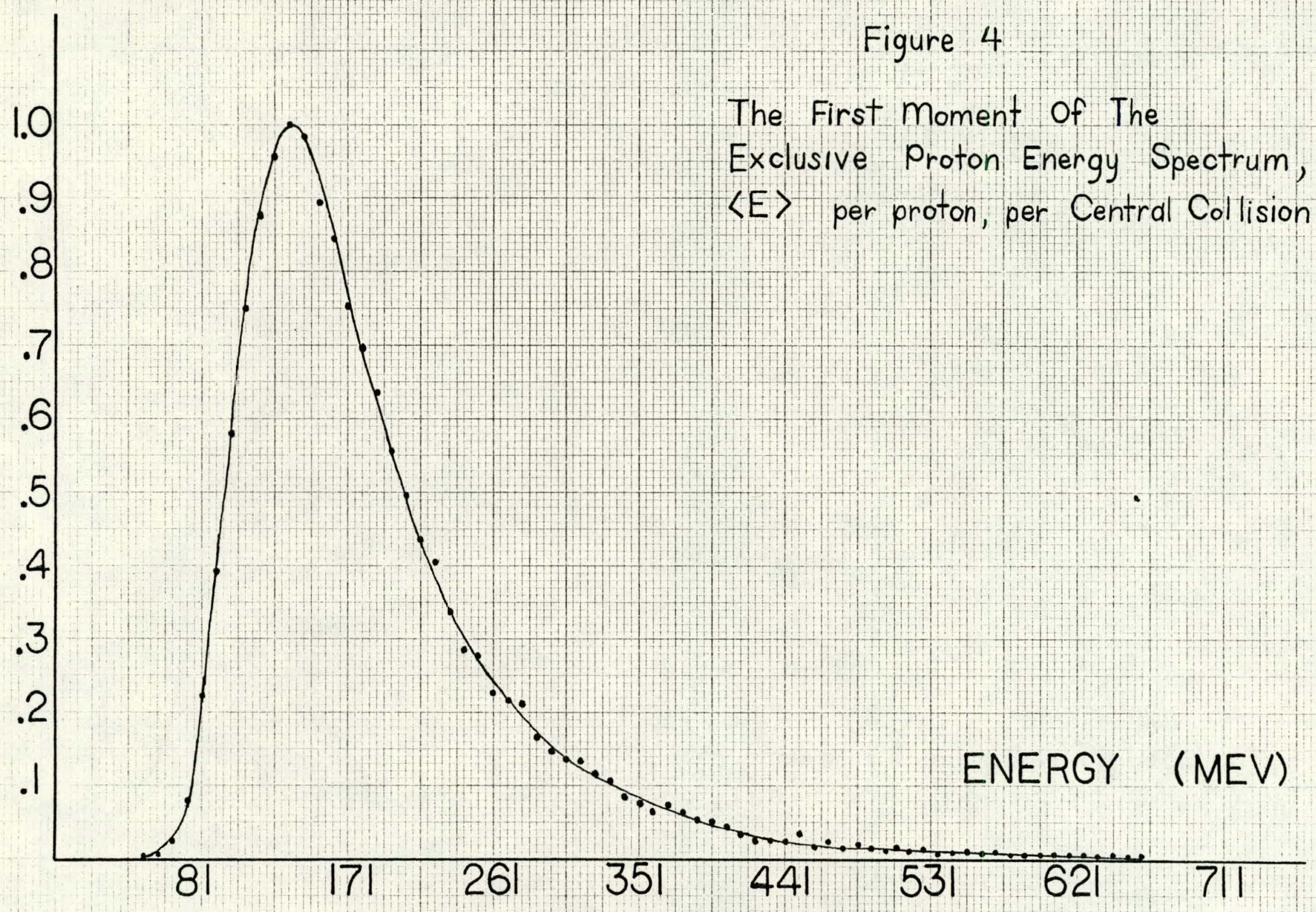


COUNTS $(X 2.1 \mathrm{~K})$

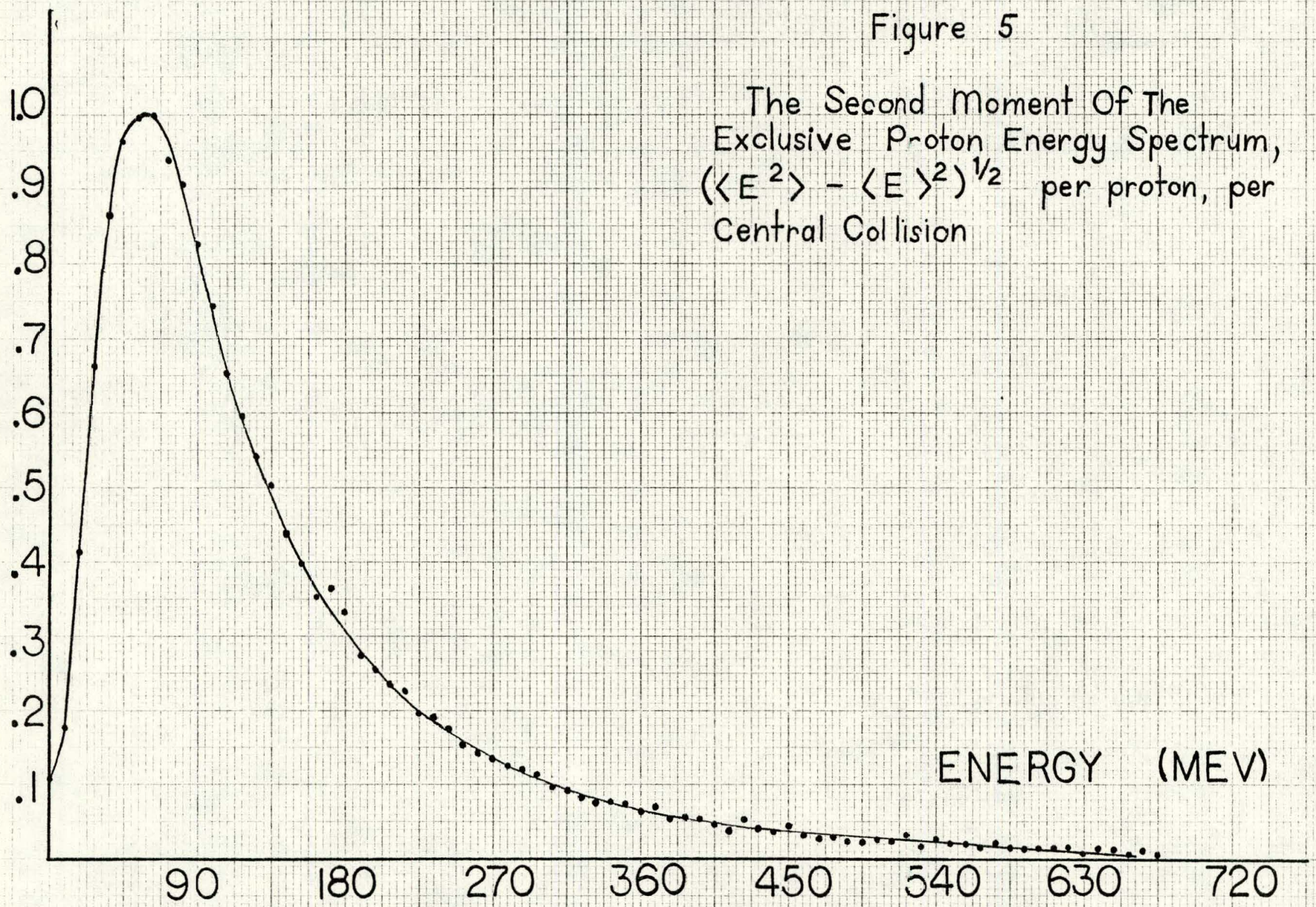




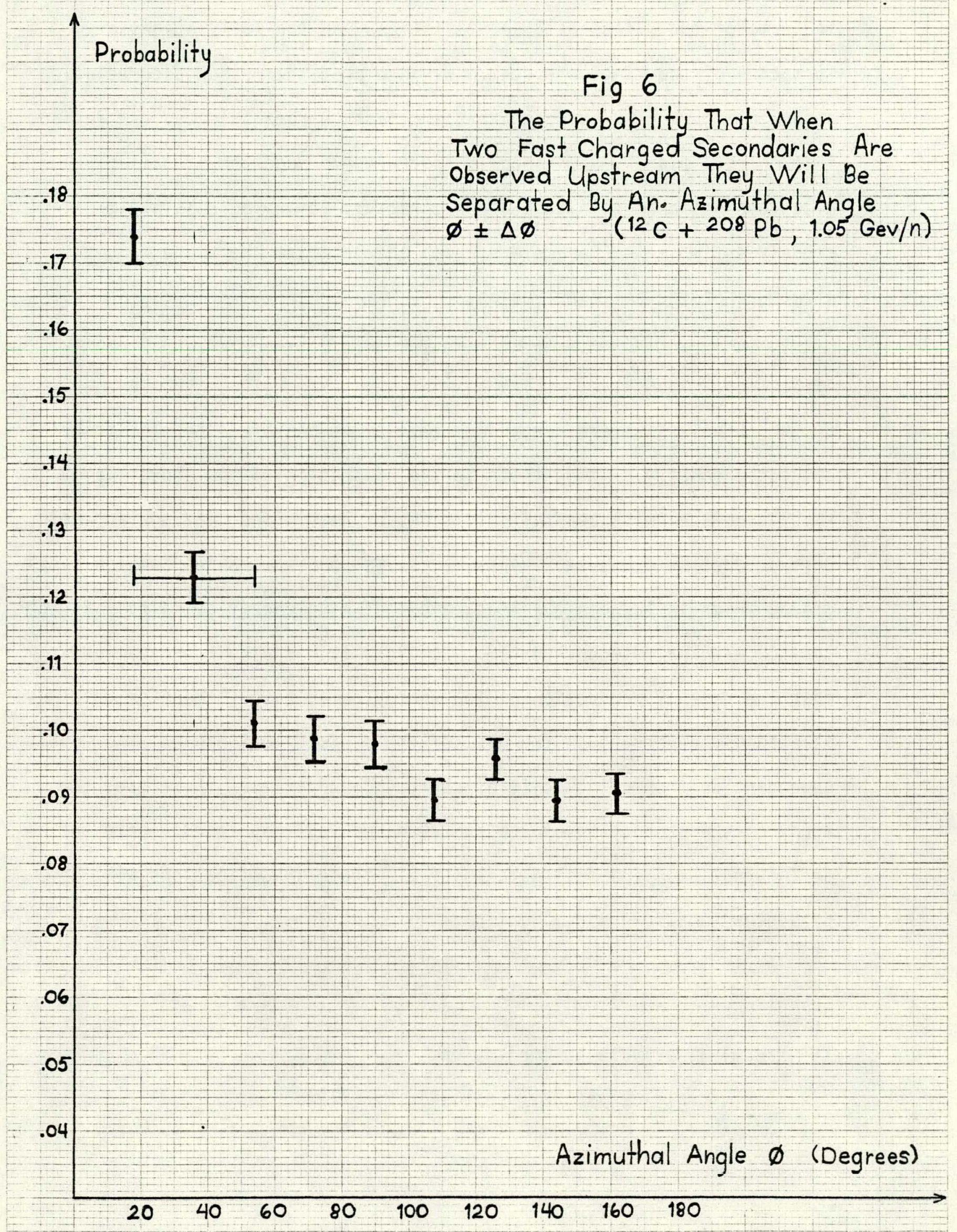


in terms of the effect of Bose statistics upon the early stages of formation of the di-pion system. We interpret the measured correlation shown in Figure 6 as evidence that the fast charges selected are in fact fast charged pions. Additionally, after the production of delta electrons has been estimated and subtracted, it may be possible to crudely estimate the parameters which characterize the space-time structure of the emitting region.

\section{Secondary Studies}

Finally, several secondary investigations are scheduled to be completed shortly. Among these is the production of low energy gamma rays associated with central collisions of heavy ions. A knowledge of this production cross section is necessary to estimate the Compton background which influences our measurement of high energy gamma rays in the lucite Cerenkov detector system. However, it has also been observed that the rate of production of low energy gamma rays ( $1 \mathrm{MeV} \leqslant \mathrm{E} \leqslant 10 \mathrm{MeV}$ ) is strongly dependent on the mass of the projectile ion used, and weakly dependent on the multiplicity of charged secondaries. Therefore, the study of the production of low energy gamma rays may itself prove to be interesting. The full details of all these investigations will be presented in a future publication.

\section{REFERENCES:}

1. Nagamiya, private communication.

2. Fung, et a1., Phys. Rev. Lett. 40,272 (1978). 
3. W. DeJarnette, T. Hallman, E. McIntyre, J. C. Walker, L. Madansky, J. Carrol1, A. Sagle and R. J. Semper, to be published in Physics Letters B.

4. The term "thin" in this context means that for a charged particle traversing a given pathlength of scintillator, the mean energy-loss is small compared to the largest energy transfer possible in a single collision with an atomic electron. The term is therefore equally applicable to a scintillation detector which is physically very thin and traversed by particles of moderate velocity or a scintillation detector of moderate thickness traversed by particles which are extremely fast. For a discussion of this subject see W. H. Barkas and M. J. Berger, NAS-NRC Publ. no. 1133, (1964), p. 103.

5. For a general formula by which the theoretical slope of the pionic energy spectrum at a given beam energy can be calculated if a thermodynamic model of pion production is assumed see M. Gyulassy and S. K. Kauffman, Phys. Rev. Lett. 40 , 298 (1978).

6. The production of alpha particles and other low $Z$ fragments is a background to the measured production of protons. This background is relatively small at the backward angles subtended by the upstream $d E / d x$ counter system.

7. S. Y. Fung, W. Gorn, G. P. Kiernan, J. J. Lu, Y. T. Oh, and R. T. Poe, Phys. Rev. Lett. 41,1592 (1978). 
IV. HIGH ENERGY GAMMA RADIATION AS A PROBE OF NUCLEAR MATTER PRODUCED IN HEAVY ION COLLISIONS*

Leon Madansky

Heavy ion collisions (HIC) are the principal source of nuclear matter at supra-nuclear densities and the physics in this region has been an object of intense experimental and theoretical investigation. It is especially important to determine empirically such parameters as local baryon density, energy density, etc. by analyzing the products of these reactions. Within the present limits of projectile energy (of the order of $2 \mathrm{GeV} / \mathrm{n}$ ) there are several directions to pursue for second generation experiments that can yield more direct evidence for the properties of nuclear matter. The emphas is in this note is placed on the measurement ${ }^{1}$ of high energy gamma rays. ${ }^{2}$

Basic programs designed to determine the spectra of $\pi^{\circ}$ and $\eta^{\circ}$ mesons via the 2-gamma decay would complement the on-going program for charged pions. For example, the absence of final state coulomb ${ }^{3}$ interaction for the $\pi^{\circ} \mathrm{s}$ may be helpful in analyzing the evolution of the hadronic process. A determination of $\eta^{\circ}$ production would be possible with the same apparatus and would provide evidence for the existence of an expanding hot region of nuclear matter in which heavier mesons may be formed in a thermodynamic process. This kind of study suggested by Glendenning et al ${ }^{4}$ must be extended to projectile energies beyond

* Submitted to LBL Heavy-Ion Collision Workshop of February 22, 1980 
$2 \mathrm{GeV} / \mathrm{n}$ to explore the important saturation region. Although the $n^{\circ}$ is one of many meson states that are of interest in this connection, its 2-gamma decay mode may be easier to detect than charged pi decay modes.

A 2-arm gamma ray spectrometer that would be suitable for the program, constructed in a modular fashion and covering a reasonably large solid angle, is described below.

A second, but more difficult, direction involves the search for direct $\gamma$ and $\gamma$ pairs. The particles produced in the HIR interact strongly so that the asymptotic final states have lost the memory of the initial high density nuclear matter. An extreme analogy would be the attempt to derive details of the interactions in the sun's core from the electromagnetic solar spectrum. Hence, a signature of the local density must have negligible interactions in the emission from hot hadronic volume induced by the HIR. Direct gamma radiation offers such a signature.

Feinberg ${ }^{5}$ has proposed a simple model to estimate the emission of gamma radiation from a hadronic medium. Although his model was constructed for energetic elementary particle collisions the application to heavy ion collisions could be equally suitable. A pion soup simulates the medium and a direct gamma ray appears from the single quantum annihilation of pions in the medium. (The strong coupling of the pions in the medium is sufficient to take up the momentum.) In the HI case one can even imagine that the pions are almost on the mass shell and can be characterized by densities $n_{+,-}$that can be estimated from cascade models. ${ }^{6}$ The number of photons expected per central collision $\mathrm{N}_{\gamma}$ in 
a $A^{40}+A^{40}$ collision is

$$
\text { where } \begin{aligned}
\mathrm{N}_{\gamma} & =\text { An } n_{+} n_{-} \cdot \sigma_{\pi^{+} \pi^{-}} \times v_{\pi} \cdot T_{\pi} \cdot v_{\text {rel }} \simeq 4 \times 10^{-3} \\
n_{ \pm} & =\text {density of } \pi^{ \pm} \simeq n_{0} \\
A & =\text { constant } \sim \text { unity } \\
V_{\pi} & =\text { relevant volume } \sim 10 \mathrm{fm}^{3} \\
\sigma_{+-} & =\text {effective cross section for annihilation } \\
& \sim \alpha \cdot 1 \mathrm{fm}^{2} \\
T_{\pi}= & \text { time interval on which high densities exist } \simeq 1 \mathrm{fm} / \mathrm{c} \\
v_{r_{e}}= & \text { rel. velocity } \sim \mathrm{c} \\
n_{0}= & \text { normal baryon density } \sim 0.2 / \mathrm{fm}^{3} \\
\alpha & \simeq \frac{1}{137}
\end{aligned}
$$

What is significant here is that the number of direct gamma rays depends on the square of the mean density reached in the central collision. The sensitive dependence on density reached is precisely the signature needed. (A medium consisting of nuclear isobars that collide, leaving a photon and two nucleons in the final state, can also model the system.) It is difficult to guess the shape of the direct gamma ray spectrum, due to the complications of strong interactions. A reasonable prediction would be the continuum extending to energies of several $m_{\pi}$ but having a different shape from the $\pi^{\circ}$ gamma continuum. A careful study in the low energy window region and the high energy tail for a hard gamma component may show significant effects of high density phenomena. Clearly the observation of direct gamma rays will depend on the shape of the spectrum and its intensity relative to the background of $\pi^{\circ}, \gamma$ rays. Figure 1 shows 


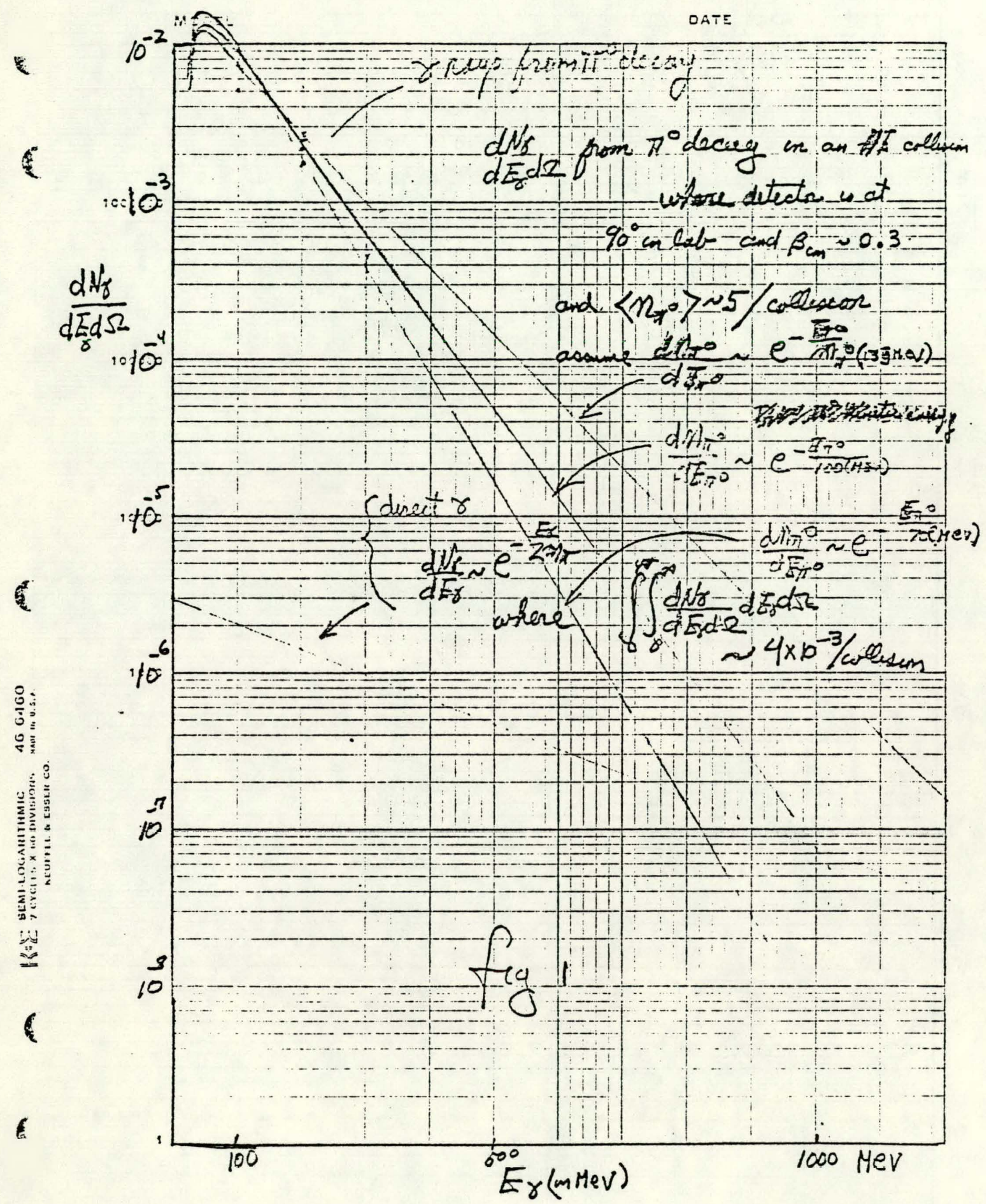




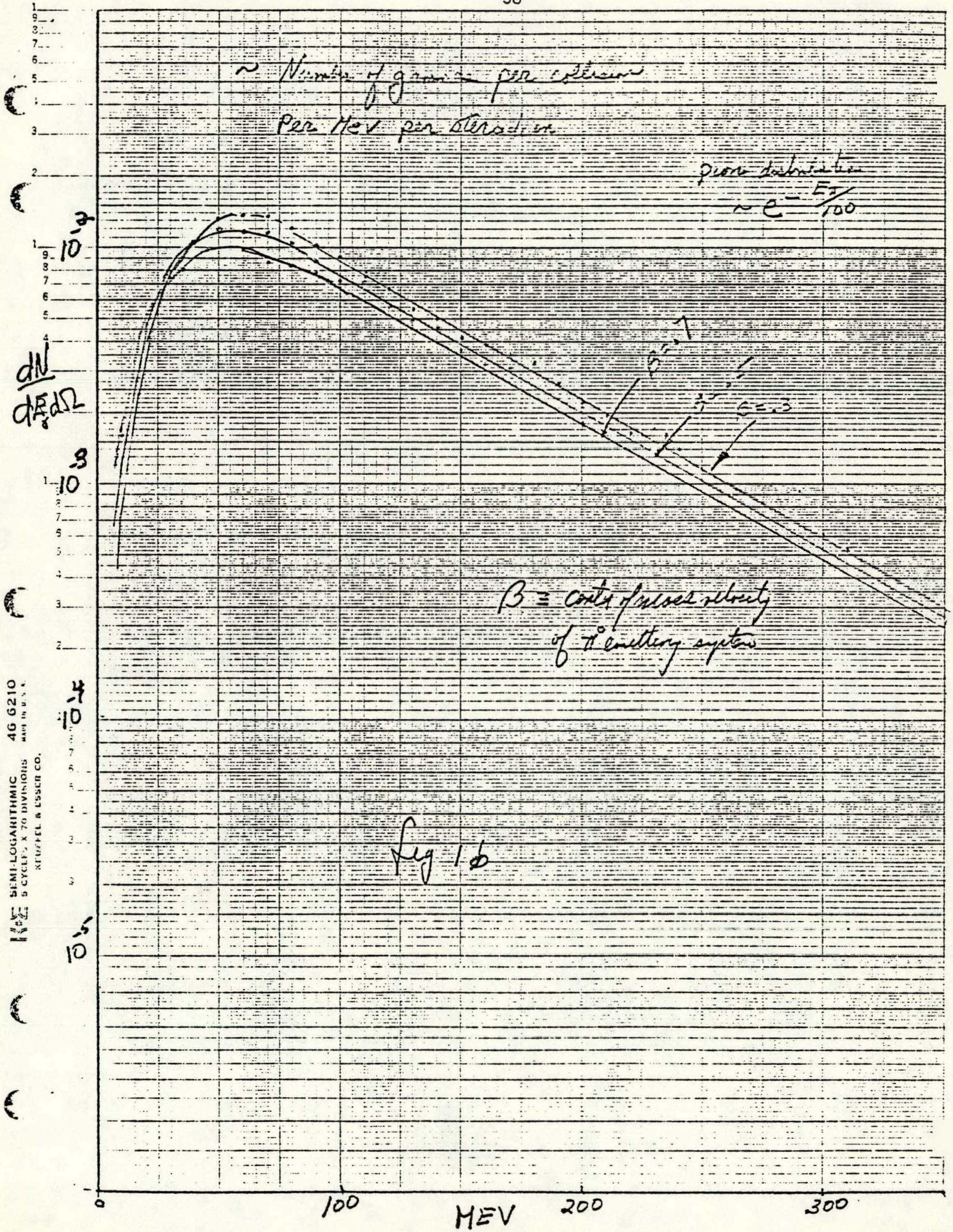


a characteristic $\pi^{\circ}$ spectrum for a reaction producing an average $\sim 5 \pi^{\circ} \mathrm{s}$ and a single gamma spectrum (a pure guess), superimposed using the estimate derived above. Unfortunately, if the estimate of $\sim 1 \%$ per central collision is reasonable for ArAr collisions at $2.0 \mathrm{GeV} / \mathrm{n}$, then such an experiment would not be possible. However, if very high density states are reached in future experiments by the onset of phase transitions or other surprises ${ }^{7}$ then the single gamma intensity should be enhanced markedly, or at least signal the onset of such phenomena (critical opalescence). The background spectrum of gamma rays from the $\pi^{\circ}$ production can be evaluated in a natural way from the results of the basic program which determines the $\pi^{\circ}$ spectrum itself.

The same annihilation process is also a source of 2 gamma rays 8 similar to the well-known process of positron annihilation; the probability, however, being down by an additional factor of $\alpha$. The background of uncorrelated gamma ray pairs from independently produced $\pi^{\circ} \mathrm{s}$ will be a severe problem, however, unless the production of $\gamma \gamma$ events per central collision is of the order of a few percent.

A third process in which the direct photon is virtual and appears as an electron-positron pair is also down by a factor of a from direct gamma production. Domokos and Goldman have reviewed the subject of Tepton pair production as a signature for the onset of a quark phase transition. ${ }^{9}$ They conclude that a sizeable enhancement can be seen when the mass of the pair lies between the pion mass and the masses of the rho and omega vector mesons $(\sim 800 \mathrm{MeV})$. This window originates from the fact that the lepton pairs from a hot hadronic medium are determined 
by vector mesons. However, the existence of a quark plasma can give rise to pairs with effective masses well below the known vector mesons. Such pairs from unrelated events such as highly asymmetric Dalitz pairs from $\pi^{\circ}$ decays, conversion of photons in the apparatus, etc. are sources of backgrounds that may, however, be sufficiently sma $11^{10}$ to make it feasible to observe the direct pair. This would particulariy be the - case if one required pairs whose effective mass was $\geq m_{\pi}$.

The directions described above, namely the determination of $\pi^{\circ}$, $\eta^{\circ}$ spectrum, the search for direct gamma radiation, and photon pairs, and the search for high effective mass electron-positron pairs seem to be independent methods of getting at measurements of hadronic densities in HIC, the key properties for the latter two being the sensitive dependence on density and the effective absence of final state interaction. Detectors designed to study these processes at presently available heavy ion energies would be equally useful when even higher energies and heavier ions (and perhaps higher nuclear densities) are avaliable in the future.

The basic gamma detector can be constructed from Pb-glass modules arrayed in two-arm assemblies. ${ }^{11}$ The intense flux of charged particles is overcome through the modular construction. In this way good events are not rejected by non-local vetos. Each module would contain an active $\mathrm{Pb}-g$ lass block $\sim 1 "$ thick to initiate a shower (wfth effictency 50\%) preceding a thicker 14 rad. length detector. This requirement reduces the effect of background from neutrons which have a small probability to interact and form a shower $(1 "=1$ rad. length or $\sim 0.1$ 
interaction lengths). A PWC placed between the shower forming block and the thick block localizes the shower and provides an accurate angular measure of the gamma ray direction. The thick $\mathrm{Pb}-\mathrm{glass}$ block absorbs the shower and together with the l" block acts as a total absorption detector. Although the resolution of $\mathrm{Pb}-\mathrm{glass}$ in the range $\sim 200 \mathrm{MeV}$ is $\sim 30 \%$, a requirement on the asymmetry parameter $x$

$$
x=\frac{E_{\gamma_{1}}-E_{\gamma_{2}}}{E_{\gamma_{1}}+E_{\gamma_{2}}} \quad \text { of } x \leq 0.1
$$

leads to a $\pi^{\circ}$ energy resolution of $\sim 5 \mathrm{MeV}$ provided that the opening angle of the 2 gamma rays can be known to $\sim 5 \mathrm{mr}$. Modules are arrayed on 2 arms (see Fig. 2) that can be shifted to cover overlapping angular ranges to determine the $\pi^{\circ}$ spectrum. The polar angle $[\theta]$ can be varied over a given range to ascertain the angular dependence of the $\pi^{\circ}$ production. Variations in opening angle $[\phi]$ covers the $\pi^{\circ}$ energy range at a fixed $[\theta]$ range.

The observation of direct gamma rays is only feasible if the spectral shape can be distinguished from the $\pi^{\circ}$ gamma continuum. In this case good resolution is necessary and $\mathrm{NaI}$ blocks should be substituted for the Pb-glass.

The detection of high mass electron positron pairs should be done with a different system. However, the 2-gamma spectrometer with an appropriate Cerenkov detector preceding each gamma module could act as a trigger for 2 relativistic charged particles that deposit sufficiently high energy in the Pb-glass or NaI to signal a high mass pair. The target region could be covered by a streamer chamber or wire chamber 
42

Boan

$-x^{2}$

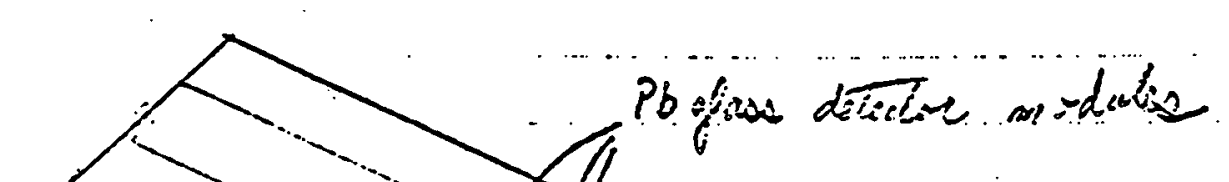

$-$

$-\cdots \cdot$

$-.$.

- -

$\cdots$
$\cdots$
$\cdots$
$\cdots$
$\cdots$

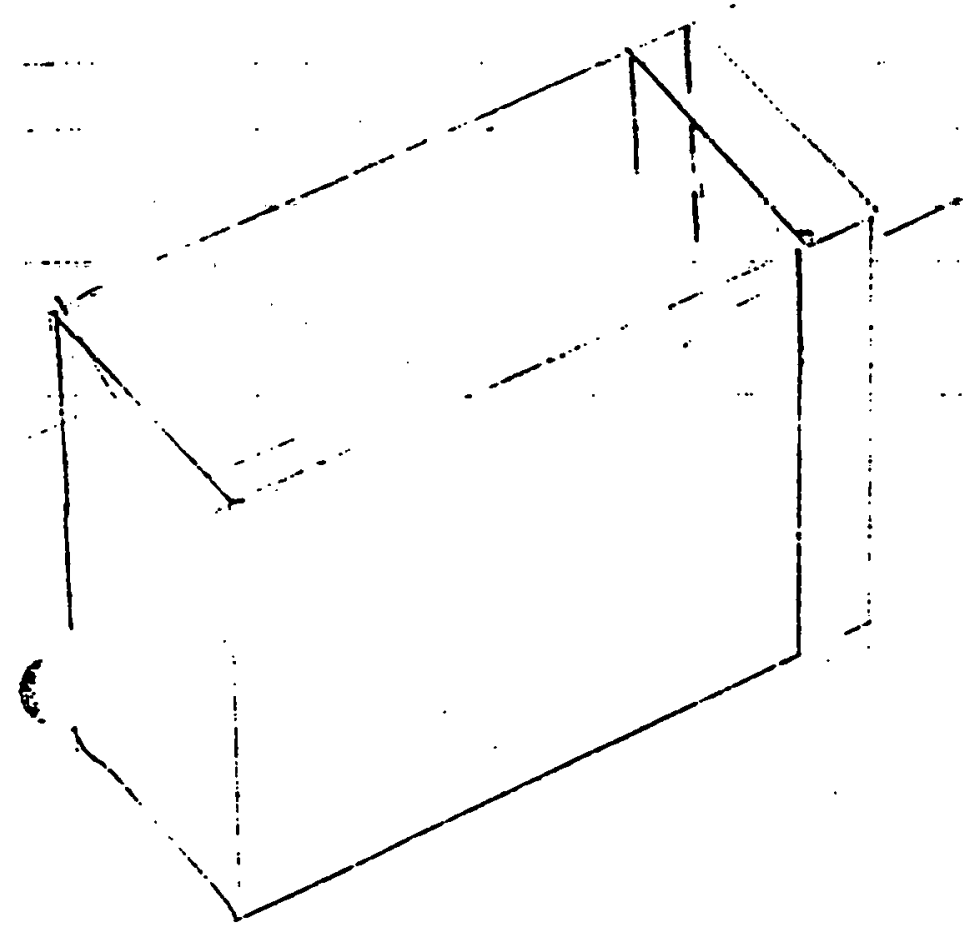

ples 9 ib

r 
system to further identify true pair and provide a direct energy measurement via the curvature in a magnetic field.

I would like to thank T. Hallman and E. McIntyre and G. Domokos for many helpful discussions.

\section{REFERENCES:}

1. W. DeJarnette et al., Bu11. Amer. Phys. Soc. 23, 959 (1978); JHU Report (unpublished) C00-3274-24, 1980.

2. Other sources of radiative effects in HIC were discussed by Kapusta, Phys. Rev. $\underline{\text { C15 }}, 1580$ (1977); and a search is in progress by Price et al., LBL.

3. K. G. Libbrecht and S. E. Koonin, Phys. Rev. Lett. 43, 1581 (1979).

4. N. Glendenning and U. Karant, Phys. Rev. Lett. 40, 374 (1978).

5. E. L. Feinberg, I1 Nuovo Cimento 34A, 391 (1976).

6. e.g., Gudima et a1., J. Phys. G: Nucl. Phys $\underline{5}, 299$ (1979).

7. S. Brodsky, SLAC-Pub-2395, Sept (1979).

8. Two photon process in pion annihilation have been proposed and carried out for $\pi^{-}$capture in nuclei. The incident pion annihilates with a virtual pion in the nucleus and, hence, is a kind of probe of the virtual pion density. T. E. O. Ericson and C. Wilkin, Phys. Lett. $57 \mathrm{~B}, 345$ (1975); J. Deutsch et a1., Phys. Lett. $80 \mathrm{~B}$. 347 (1979).

9. G. Domokos and J. I. Goldman, Quark Matter Diagnostics, JHU-HET-8004, May 1980.

10. An experiment to study background of this type has been approved by the Bevatron PAC and is planned for execution in the fall of 1980 . 
11. Many gamma spectrometers have been constructed. A most suitable example is described by J. D. Bowman et al., IEEE Transactions on Nuc. Ser., Vor. NS25, 344 Feb (1978). 
V. ALPHA-ALPHA COLLISIONS AT ULTRARELATIVISTIC ENERGIES

Leon Madansky

An experiment to study the physics of alpha-atpha collisions at $15 \mathrm{GeV} / \mathrm{n}$ at the intersection storage ring (ISR) at the CERN laboratory in Geneva for the latter part of July is projected. A European collaboration with an established experiment, R807, (spokesman: Mike Albrow) has been kind enough to accept the participation, for this particular run, of a University of Pennsylvania group under the direction of Sherman Frankel and Leon Madansky from Johns Hopkins. The R807 experiment is normally devoted to the study of hadrons from $p-p$ interactions at very high energy with the use of elaborate spectrometers with particle identification. The detection system in addition is equipped with reasonably large area calorimeters for the detection of future jets. Preliminary studies by Frankel et al. (internal report to $R 807$ group) have shown that these calorimeters are sufficiently segmented to signal the interaction of all the nucleons in each of the beams so that a central collision would be characterized by multijets $(\sim 4)$ in each of these calorimeters. We expect to use these calurimeters as a primary trigger to select central collisions and study the correlated hadronic production in the spectrometer as a whole. The possibility of new or unexpected phenomena that may show up in these collisions could appear as a marked increase in wide angle multiplicities or changes in the nature and spectra of the hadronic components. The analysis will be carried out in a computer facility in the Physics Department of the University of Pennsylvania. Some preliminary results should be available before the end of the calendar year 1980. 
VI. NEW PRECISION PIONIC MASS MEASUREMENT AND MU-NEUTRINO MASS VALUE FROM PIONIC ATOMS

Y. K. Lee, A. J. Caffrey and R. Levin, in collaboration with C. S. Wu (Columbia) and D. C. Lu (Yale).

High resolution studies of pionic $X$-rays using a bent-crystal spectrometer was carried out by placing the $x$-ray target directly next to the pion-production target at the Nevis synchrocyclotron. In our previous Progress Report ${ }^{1}$ we described the observation of the low-yielding fine-structure component $\pi-T i(5 f-4 d)$, providing an experimental measurement of the relativistic fine-structure splitting in this pionic atom. The experimental arrangement was published, ${ }^{2}$ and consisted of a modification of the experimental approach used by Marushenko et al., 3 and a spectrometer representing a new technique of crystal bending developed by Dr. Lu at Yale.

In the previous reports 1,2 it was pointed out that the plontc $X$-rays were measured relative to the gamma ray 1 ine in ${ }^{99}$ Mo which had an energy within $\frac{1}{2} \mathrm{keV}$ from that of the pionic $X$-ray. The precision of the present experiment exceeded the precision of the energy measurement of the gamma ray line available at that time. In order to make maximum of the current data, a new measurement had to be carried out for the energy of the reference line at the National Bureau of Standards. Using the measurements of the pionic $X$-ray energies of the $4 f-3 d$ transition in $\pi-P$ and the $5 g-4 f$ transition in $\pi-T i$, we have deduced the $\pi^{-}$mass value to an accuracy of $\pm 6.4 \mathrm{ppm}$. These measurements consist 
of directly measuring the small energy differences between these $X$-rays and an energy reference 1 ine, the $40583.36 \mathrm{eV}$ gamma-ray in ${ }^{99}$ Mo whose energy was measured separately with a double flat crystal spectrometer at NBS. ${ }^{4}$ Sample data of the $\pi^{-}-P X$-ray and the reference $\gamma$-ray are shown in Figure 1. Our new measured value of $\pi^{-}$-mass, on the basis of the new wavelength and energy standards of $\mathrm{NBS}^{5}$ is

$$
m_{\pi^{-}}=139567.1 \pm 0.9 \mathrm{keV} .
$$

In comparison with our new measurement, the three best previous measurements are:

(1) for $\pi^{-}, m_{\pi^{-}}=139566.7 \pm 2.4 \mathrm{keV}$ by Marushenko et al.$^{3}$

(2) for $\pi^{-}, m_{\pi^{-}}=139568.6 \pm 2.0 \mathrm{keV}$ by Carter et a ${ }^{5}$.

(3) for $\pi^{+}, m_{\pi^{+}}=139565.8 \pm 1.8 \mathrm{keV}$ by Daum et al.$^{7}$

The last $\pi^{+}$mass value was deduced from measuring the $\mu^{+}$momentum in $\pi^{+} \rightarrow \mu^{+}+\nu_{\mu}$ decay, assuming the $\nu_{\mu}$ mass to be zero, and using the mass of $\mu^{+}$, known to $\sim 2.5 \mathrm{ppm}^{8}{ }^{8}$ Our new value is in excellent agreement with the average of the three, assuming $m_{\pi^{+}}=m_{\pi^{-}}$.

Alternatively, if $m_{\pi^{+}}=m_{\pi^{-}}$, our new mass value, combined with measured $\mu^{+}$momentum of $P_{\mu}=29.7877 \pm 0.0014 \mathrm{MeV} / \mathrm{c}$ in $\pi^{+}$decay, ${ }^{7}$ leads to a new upper bound for the $\mu$-neutrino mass, $m_{v}{ }^{2}=0.078 \pm 0.119 \mathrm{MeV}^{2}$, and $m_{v}<0.5 \mathrm{MeV}$ ( $90 \%$ confidence).

The present results and further analysis are submitted to the 7 th International Conference on Atomic Physics. ${ }^{9}$ 


\section{REFERENCES :}

1. "Nuclear Moments and Nuclear Structure," Annual Progress Report to DOE, Leon Madansky and Yung Keun Lee, (C00-3274-23) 1979, p. 25.

2. L. Delker, G. Dugan, C. S. Wu, D. C. Lu, A. J. Caffrey, Y. T. Cheng, and Y. K. Lee, Phys. Rev. Letters, 42, 89 (1979).

3. V. N. Marushenko et al., Pisma, Zh, Eksp. Teor. Fiz. 23, 72 (1976).

4. Private communication, Drs. R. D. Deslattes and E. G. Kessler, Jr., National Bureau of Standards.

5. E. G. Kessier, Jr., R. D. DesLettes, A. Henins and W. C. Sauder, Phys. Rev. Letters $\underline{40}, 171$ (1978).

6. A. L. Carter et a1., Phys. Rev. Letters 37, 1380 (1976).

7. M. Daum et al., Phys. Rev. D20, 2692 (1979).

8. Particle Data Group, Phys. Letters 75B, 1 (1978).

9. Submitted to the 7 th International Conference on Atomic Physics, M.I.T., August 4-8, 1980. 
VII. THE NEUTRON AND GAMMA-RAY CORRELATION IN THE $\pi^{-}$-CAPTURE IN ${ }^{165}$ HO AND ${ }^{181} \mathrm{Ta}$

R. Levin., E. McIntyre, Y. K. Lee and L. Madansky

In the past few years the physics of the $\pi^{-}$-capture has been pursued from a new angle using medium to heavy nuclei. This is partly due to the fact that the relatively new heavy-ion physics provided interpretation to the nuclear processes involved in the highly excited nuclear states following the $\pi^{-}$-capture. On the other hand, the data from the study of the $\pi^{-}$-capture will be valuable in the interpretation of dissipation of energy and angular momentum in energetic heavy ion collisions.

We have started the current series of experiments in order to study the initial nuclear processes of capture and the nature of the nuclear states following the capture. Our experiences with the heavy ion physics was to be applied to the study of the evolution of nuclear states, particularly neutron emitting states, and finally to the study of the de-excitation mechanisms in the residual nuclei.

Our preliminary work on the neutron-gamma ray coincidences following the $\pi$-capture in ${ }^{165_{\mathrm{Ho}}}$ and ${ }^{181} \mathrm{Ta}$ has been already described in the previous Progress Report. ${ }^{1}$ The past year has been spent mainly in upgrading the experimental arrangement so that the counting statistics can be improved by two orders of magnitude and moving the experiment to the new stopped-pion channel (M13) at the Tri-University Mason Facility (TRIUMF) of the University of British Columbia. The 
experiment (TRIUMF Exp. No. 145) is scheduled to be staged in October of this year.

The negative pion is captured mostly from $4 \mathrm{f}$ orbit in mediumheavy nuclei by a pair of correlated protons and neutrons. The nucleons seem to share the rest mass of pion and move back-to-back. These nucleons seem to undergo various degrees of rescattering before they emerge from the nucleus. One or both of them can be absorbed in the nucleus initiating a series of collisions and excitations before the nucleus reaches the equilibrated nuclear state. The excited nucleus subsequently evaporates mostly neutrons, and finally populates the ground state rotational bands of the residual nucleus in a fashion similar to yrast cascades in heavy ion physics. ${ }^{2}$

With twelve large scintillators we will measure neutron-neutron correlations, and by use of a large Ge(Li) detector we will also measure the neutron-gamma ray correlations. Due to the large multiplicity of radiations and the large number of counters we expect considerable triple coincidences, among three neutrons, or two neutrons and one gamma ray. When the neutron spectra is taken in coincidence with the discrete gamma rays detected by a $G(L i)$ counter, one has the information about the final isotope, and therefore the neutron multiplicity. The event will also be tagged by the angular momentum of the residual nuclei, and this is especially important in the study of the angular momentum dissipation of the de-excitation process. Since the alignment of the nucleus is expected to survive the thermal neutron emission and statistical gamma-ray cascade, the angular correlation between the pre-equilibrium neutrons and the gamma ray gives us information 
concerning the angular momentum dissipation, complementary to the gamma ray multiplicity analysis.

In the neutron-gamma ray correlation, the elucidation of the mechanism of population of very high-spin states is perhaps the most immediate goal of our experiments. Several conjectures exist in this regard, ${ }^{3}$ but a detailed study of the mechanism is a very significant step toward understanding the momentum and angular momentum dissipation in this class of reaction, including those encountered in the energetic heavy-ion collisions.

\section{EXPERIMENTAL ARRANGEMENT}

The experimental arrangement consists of the neutron time-of-flight system using the stopped pion signal from the pion telescope as timezero. The system has twelve large plastic scintillators mounted in an arch to measure angular correlation, and all scintillators have a flight path of 5 feet. A large volume $G(L i)$ detector is used in coincidence with neutrons to identify the discrete gamma rays of the final isotope. A CAMAC-based data-retrieval system is used in connection with the software "Multi", developed at the Fermi National Laboratory and the TRIUMF.

Each neutron counter consists of an NEII0 plastic scintillator of $25^{\prime \prime} \times 6^{\prime \prime} \times 3$ " in dimension, viewed by a pair of " 5 " phototubes on both ends in a position-sensitive mode. The phototubes are coupled by means of 8 " light pipes which are shaped to transmit preferentialiy the oncescattered light from the scintillator as a good compromise for a good timing and good light collection. Calibrated against the cosmic ray 
53

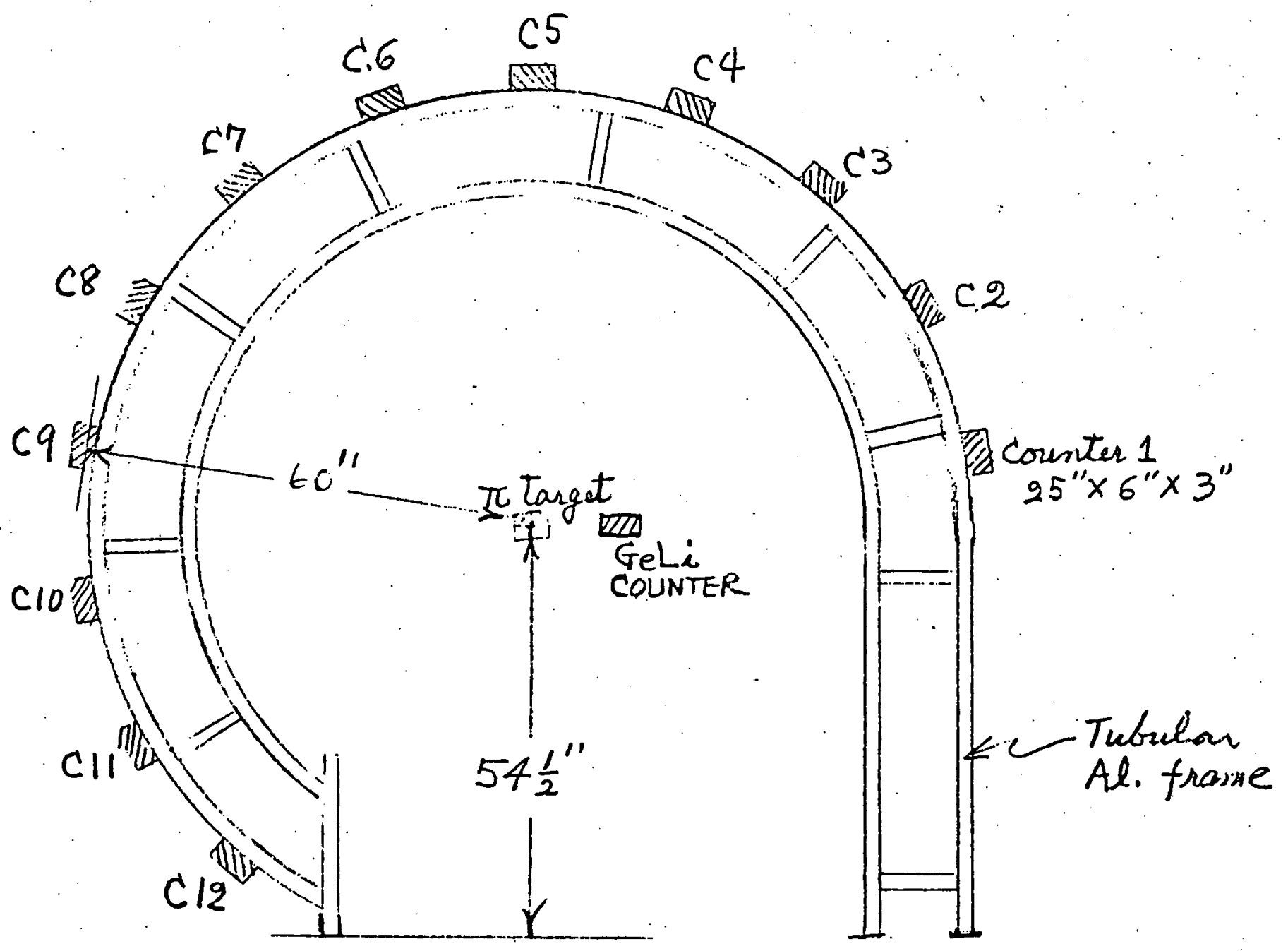

Figure 1. Geometry of the neutron and gamma ray counters, in the plane perpendicular to the pion beam axis. 
muons, each detector is capable of timing resolution better than 600 psec, and space resolution of $2 "$ when operated in position-sensitive mode.

The neutron counters are mounted on a cylindrical aluminum frame in such a way that the axis of the frame as well as the axis of each counter is parallel to the axis of the pion beam as shown in Figure 1. The neighboring counters are separated by at least $18^{\prime \prime}$ in order to reduce scattering between counters, and subtend an angle of $18^{\circ}$ with respect to the pion target. There are three pairs of detectors at $180^{\circ}$ to each other, and this arrangement was intended to increase the statistics for triple coincidences, because the back-to-back emission of neutrons is still the dominant mode. The aluminum frame was made of hollow tubing in order to minimize the scattering, and any part of the main frame is at least 2" away from the scintillators. There is provision to reduce the flight path to $4^{\prime}$ from $5^{\prime}$ just in case a larger counting statistic becomes mandatory.

In order to run each neutron counter in a position-sensitive mode, each phototube at each end of the counter is provided with a time-to-digital converter (TDC) via constant fraction (CF) discriminator. The dynode output from each phototube is also processed by individuat analog-to-digital-converters $(A D C)$ because the efficiency of the neutron counters depends on the discrimination level.

As shown in Figure 2, the time-zern signal, or the start signal for the TDC, is derived from the pion-stop signal. One nice feature of the M13 channel at the TRIUMF is the possibility of generating a veto signal for muons and electrons by timing the pion stop signal with respect to the RF structure. Since we are expecting more than $10^{5}$ 


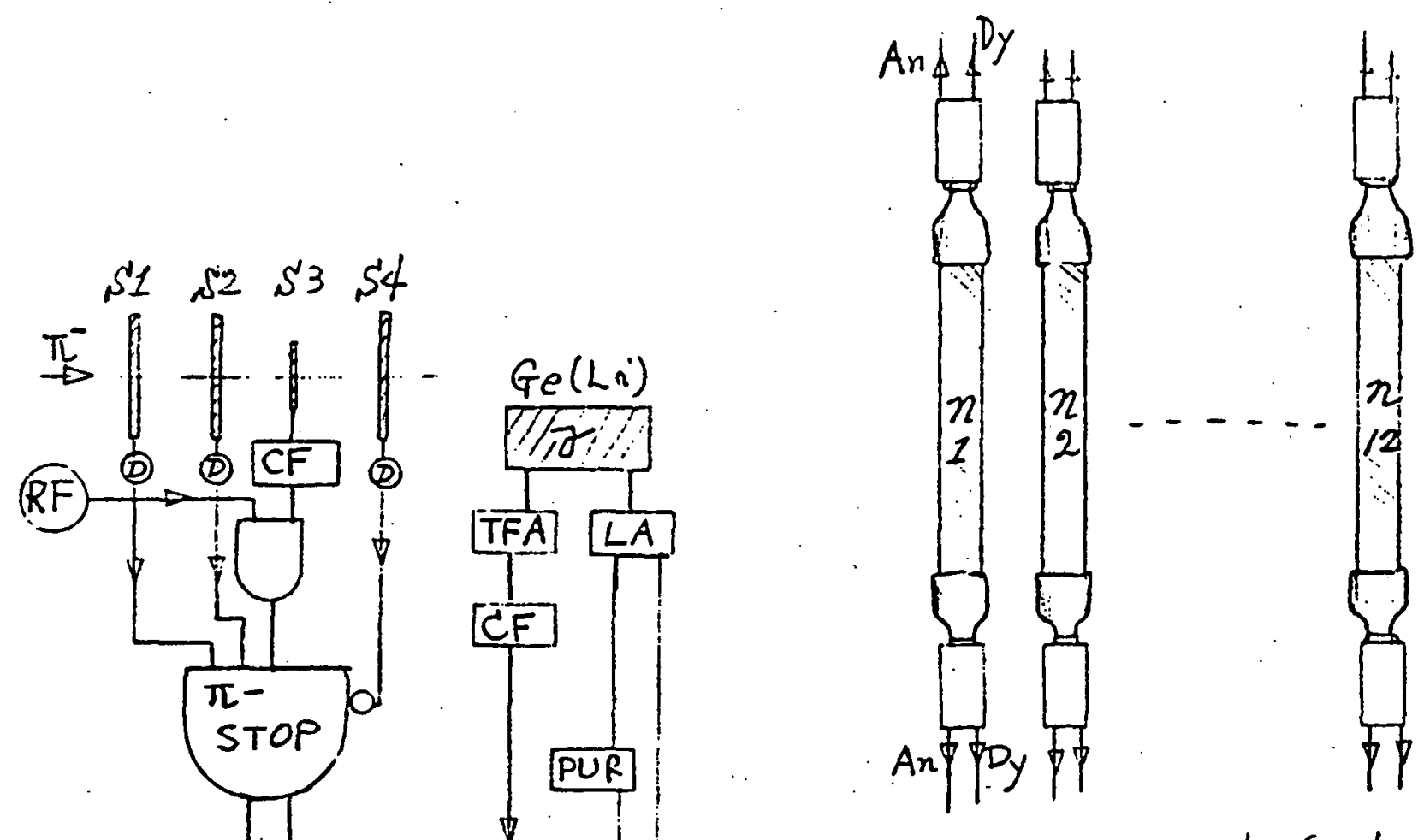

Orle end of n's

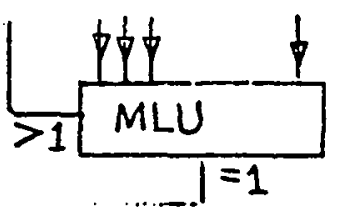

An: Anode

Dy: Dynode

$C F:$ Constant fraction discr. $D$ : discriminator $\delta$ : delay PUR: Pile up reject (' MLU: Majori't Logit TFA. Trine filtering Amp Figure 2. Electronics and logic diagram for the pion stop, 12 neutron counters, and gamma ray counter. 
stopped pion per second it was decided to gate the start signal by the output of the multiplicity logic unit and/or coincidence gamma ray signal as shown in Figure 2. The start signal is delayed 100 ns while the neutron time-of-flight signals and gamma ray signals are processed to derive either a neutron-neutron or a neutron-gamma ray coincidence signal as gates. The ultimate time-zero is obtained by the thin scintillator (53) in the pion telescope, and should be delayed by cable so that it arrives last in the quadruple logic units (QLU) termed "Master Gate" in Figure 2, in order to strobe the QLU. All of the 24 stop signals should be correspondingly delayed.

\section{DISCUSSION}

By studying the neutron and gamma-ray correlations following $\pi^{-}$ captures in medium heavy nuclei, we are interested in understanding the complex nuclear deexcitation and the nature of nuclear states involved. We are in particular interested in the mechanism of the angular momentum transfor, or dissipation, by determining the spins of the nuclear states through gamma ray spectroscopy. In this connection we have also carried out parallel studies using heavy ion beams and measuring neutron-neutron correlations gated by gamma ray multiplicity, as reported elsewhere in this Progress Report.

In our past measurement of neutron-gamma ray correlation following $\pi^{-}$-captures at the Nevis Synchrocyclotron, we observed interesting correlations among the neutron spectra, gamma ray spectra, and neutron multiplicity. The data were discussed in our previous Progress Reports. 1 


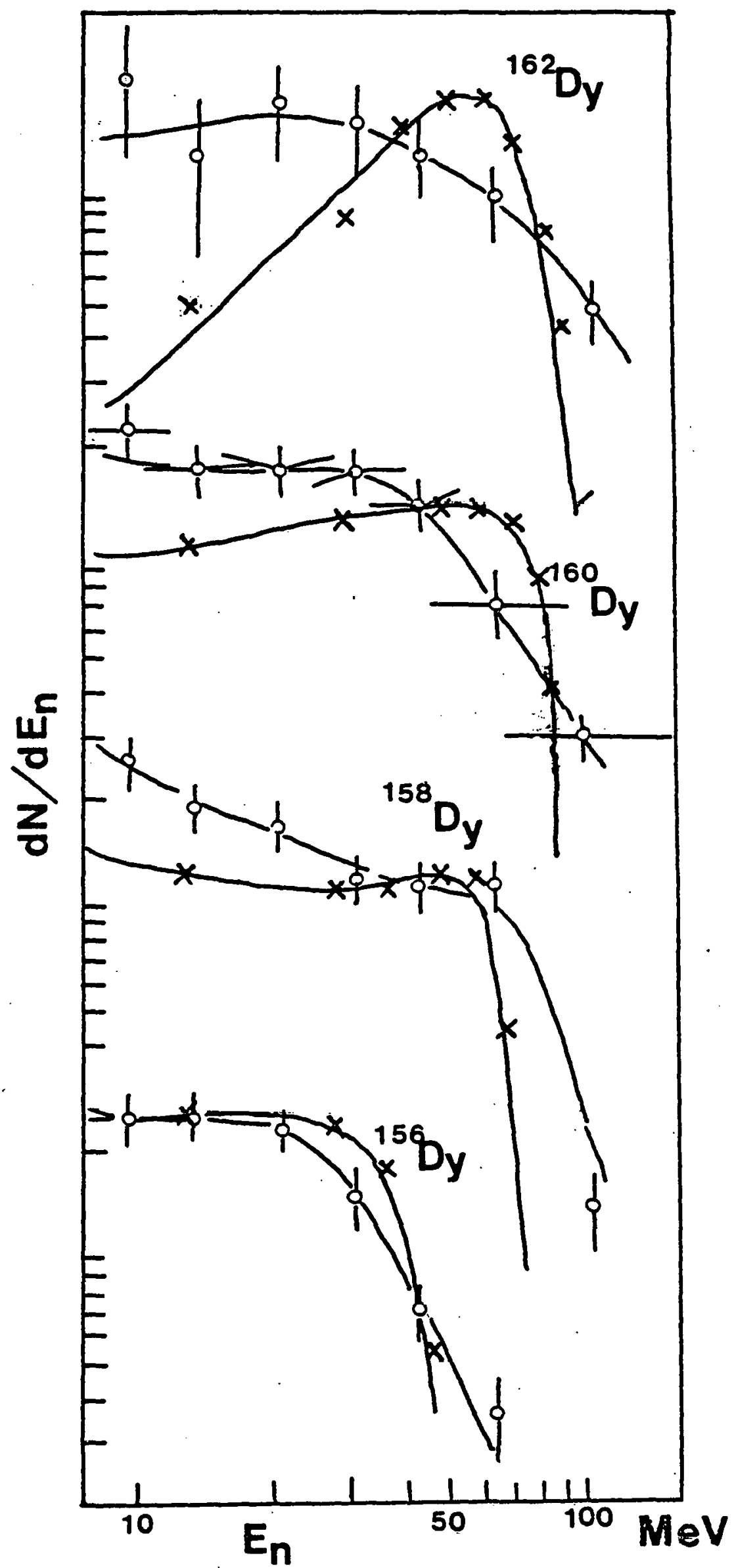

Figure 3. Neutron time-of-flight spectro in coincidence with $4^{+}$to $2^{+}$ gamma ray transitions with $165_{\mathrm{Ho}}$ target. Experimental data are shown in open circles and the exciton model prediction in crosses for various final isotopes. 
Some of the effect of the neutron multiplicity on the neutron spectra coincident with discrete gamma rays can be understood on a simple model based on the exciton model. In Figures 3 and 4 , the neutron spectra with $165_{\mathrm{Ho}}$ and ${ }^{181} \mathrm{Ta}$ are reproduced, respectively, with some of the new calculation of neutron spectra superimposed. The calculations consider the probability of emission of one pre-equilibrium neutron from each branch due to the two primary nucleons, and find the resulting emitted neutron energy spectra and residual nuclear excitation. The nucleus is assumed to have reached statistical equilibrium at this point, and the results of the pre-equilibrium calculations are passed to a particle evaporation code in order to predict further neutron emission. ${ }^{4}$ The results of these calculations are the daughter isotope yields and neutron spectra for the daughter isotopes. The calculated spectra in the two figures are similar to the coincident neutron spectra, but are not directly comparable since the experimental spectra were taken in coincidence with gamma rays connecting nuclear states of known spin and parity, while the pre-equilibrium calculations did not include any spin dependence.

The data of our experiments at the Nevis Synchrotron were preliminary, and we did not achieve the level of statistics to definitely correlate, for example, the neutron spectra to the spins of the nuclear states, or to measure the angular distributions. However, strong correlations were observed as seen in Figures 3 and 4 . We have also seen the effect of the coincident neutron energy on the lineshape of the quadrupole splitting of the $\pi^{-}-\mathrm{Ta}(5 \rightarrow 4) X$-ray, as seen in Figure 5 .

Because of the unexpected nature of the observation various 


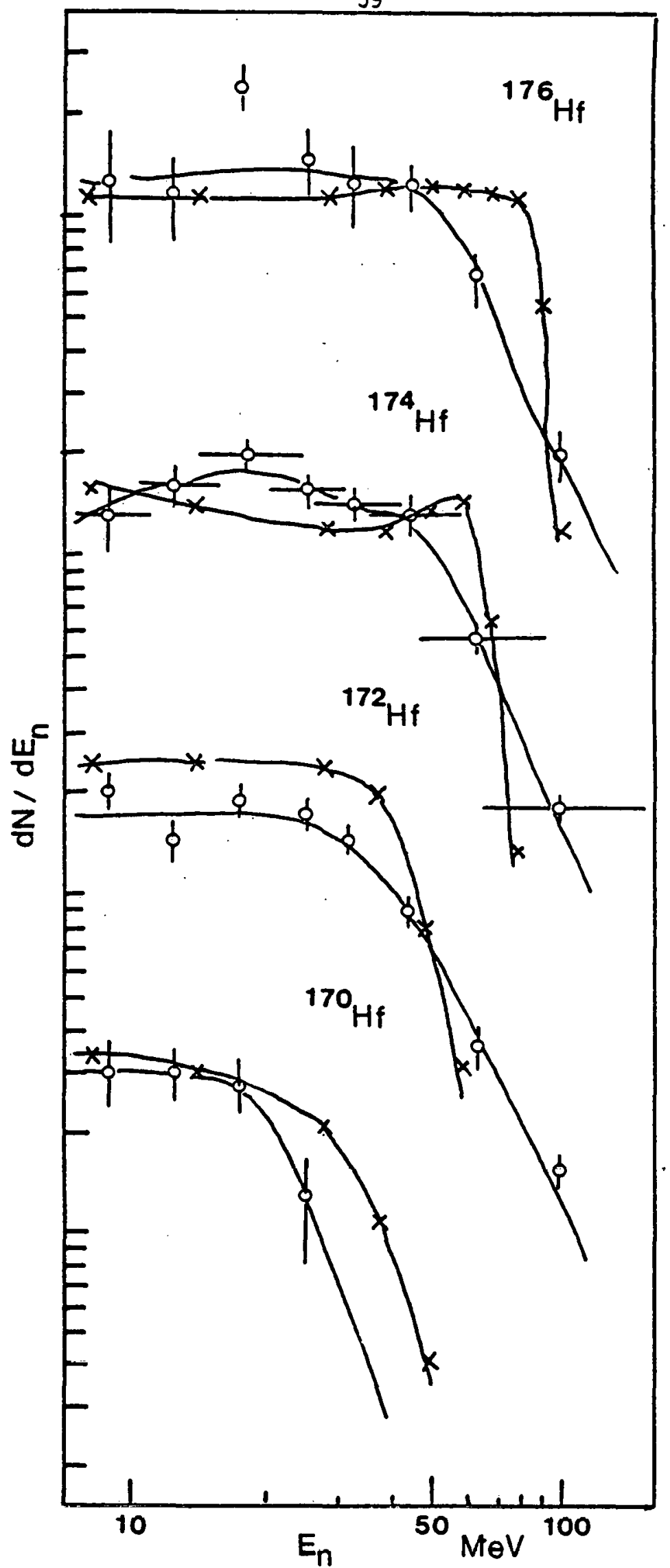

Figure 4. Neutron time-of-flight spectra in coincidence with $4^{+}$to $2^{+}$garma ray transitions with 181 Ta target. Experimental data are shown in open circles and the exciton model prediction in crosses for various final isotopes. 
internal checks were done even with a complete change in data retrieval system and timing characteristics. The only interpretation one can propose at this stage is that both the neutron spectra and the $X$-ray lines depend on the final location of the pion in the nucleus at the moment of capture. These data are preliminary in character, but show significant correlation, and provide a strong motivation to pursue our planned experiments at the TRIUMF

\section{REFERENCES:}

1. Nuclear Moments and Nuclear Structure, Annual Progress Report to DOE, Leon Madansky and Yung Keun Lee (CO0-3274-23) 1979, p. 43.

2. R. Beetz et al., Z. Phyzik, A286, 215 (1978) and the references quoted therein.

3. E.Gadiali et al., Nucl. Phys. A256, 414 (1976); M. P. Locher et al., Helv. Physica Acta 49, 123 (1976); A. S. I11jinuv enl al., Nuc1. Phys. A265, 573 (1976).

4. Variation of codes discussed in J. R. Wu et al., Phys, Rev, . C.1h, 1812 (1977). 


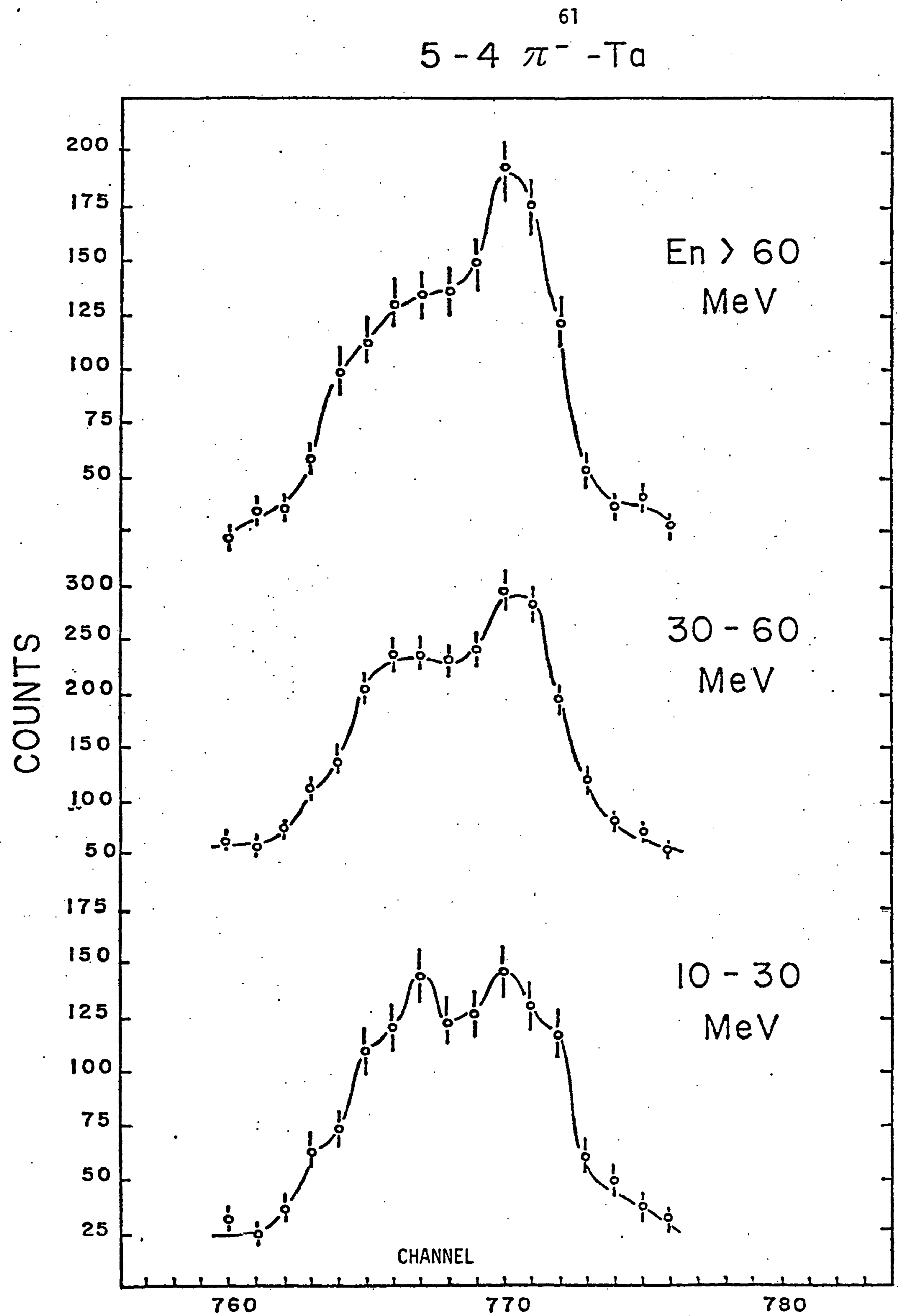

Figure 5. The quadrupole line broadening of the $\pi^{--}-T a(5 g \rightarrow 4 f)$ transition-in coincidence with neutrons in three different energy ranges. 
VIII. THE NEUTRON-NEUTRON AND THE NEUTRON-GAMMA RAY CORRELATION IN THE

$$
\text { REACTION }{ }^{12} \mathrm{C}+{ }^{158} \mathrm{Gd}
$$

R. Levin, A. J. Caffrey, Y. K. Lee and L. Madansky, and D. A. Goldberg (University of Maryland)

In the past few years, the phenomena of particle and gamma ray emissions in the damped heavy-ion collisions have been a subject of intensive studies, ' and the deviations from the expectations based on the compound nuclear states following fusion-like collisions have been found, ${ }^{2}$ as the projectile energy was increased beyond $10 \mathrm{MeV} / \mathrm{AMU}$. The presence of non-equilibrium processes in heavy ion induced reactions has serious implications on the energy and angular momentum dissipations, and many models such as pre-equilibrium process, or the prompt particle emission process, have been advanced.

Our main interest in this line of research is its implication in the understanding of the nuclear processes following the $\pi^{-}$-capture in medfum heavy nuclef. As described in another part of this progress report we are concerned with the neutron-neutron, and neutron-gamma ray correlation during the de-excitation of nuclear states following the $\pi^{-}$-capture. The present questions on the nature of highly excited nuclear states and de-excitation processes are common to both the heavy ion process and the $\pi^{-}$-capture process. The present experiment with the heavy ion beam was undertaken as a preliminary step toward a more ambitious project with the $\pi^{-}$-beam at the TRIUMF accelerator.

Evidence for the pre-compound emission of neutrons from fusion-like 
reaction was not unequivocal until recently. The confusion was caused by the products of the projectile fragmentation and the deep inelastic scattering. Sarantites and coworkers ${ }^{2}$ reported the pre-equilibrium nature of the deexcitation from the fusion-like reaction of ${ }^{12} \mathrm{C}+{ }^{158} \mathrm{Gd}$ on the basis of saturation in the gamma-ray multiplicity as the projectile energy reached $125 \mathrm{MeV}$. The saturation of the gamma ray multiplicity is attributed to the onset of emission of one pre-equilibrium neutron at this bombarding energy, which may carry a large angular momentum and thus limit the balance of angular momentum left to the product nuclei. They further deduce the angular momentum carried by the first pre-equilibrium neutron to be of the order of $3 \mathrm{~K}$ at $145 \mathrm{MeV}$ bombarding energy and such angular momentum value rapidly would rise above this bombarding energy. This picture of pre-equilibrium decay in which the first neutron carries a large energy and angular momentum, and the residual nuclei decay by statistical process can be examined if we measure the correlation between the energy of the first neutron and the energy of one of the statistical neutrons. If the pre-equilibrium neutron indeed carries a rather large angular momentum, then the neutron-neutron angular correlation will be an effective probe of such effect.

We also attempted to detect the correlations of two neutrons originating from the indistinguishability of the pair of nucleons. A possibility of measuring the lifetime for excited nuclei in the interval $10^{-18}$ to $10^{-22}$ seconds exists when certain criteria are met, including the instrumental resolution of the energy of the nucleons. ${ }^{3}$ Such measurements are now proving to be a powerful method of determining the 
space-time correlation wherever identical particles are emitted by highly excited nuclear states, and have been recently applied to multipion emitting states. 4

The coincidence measurements between neutrons and neutrons, and neutrons and gamma rays in this situation has been rather rare in the past because of the experimental difficulty, mainly due to low efficiency of neutron counters and the background problems. A major improvement is necessary to increase the efficiency of the neutron detection over conventional methods and to provide an effective trigger to reduce the large background. In the past year we built a time-of-flight system for neutrons consisting of three large plastic scintillators in positionsensitive mode, and a gamma-ray multiplicity trigger with an efficient neutron discrimination. The preliminary runs at the University of Maryland cyclotron. indicate that the system is capable of multi-neutron correlations with a trigger based on the gamma-ray multiplicity counters. The cyclotron was not capable of providing more than 20 particle-pamp. of ${ }^{12} \mathrm{C}$ before the accelerator was closed down in June 1980, and we were not able to incorporate a $\mathrm{Ge}(\mathrm{Li})$ counter for neutron gamma-ray coincidences, but the system is now thoroughly tested for a future experiment at a larger facility with beam energy exceeding $10 \mathrm{MeV} / \mathrm{AMU}$.

\section{EXPERIMENTAL ARRANGEMENT}

The experimental apparatus consisted of the neutron time-of-flight (TOF) system made of plastic scintillators and the gamma ray multiplicity counters. A large $\mathrm{Ge}(\mathrm{Li})$ detector was available but was not included 


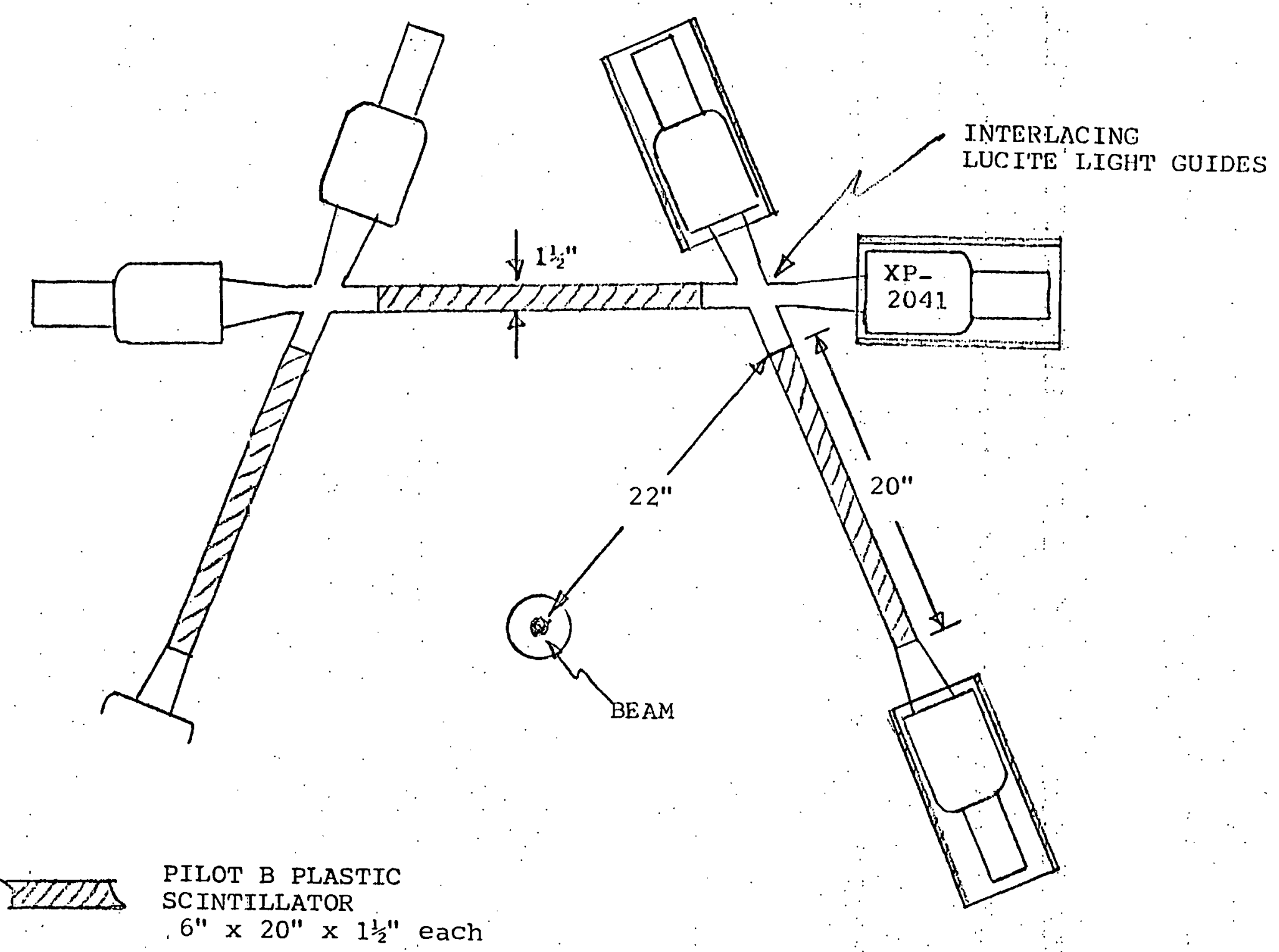

Figure 1. Neutron time-of-flight arrangement with three position sensitive scintillators. The counters are in a plane perpendicular to the beam axis. 
in the correlation study because of the low beam current. The Ge(Li) detector was useful only as a monitor. The data including the timespectra from six phototubes and the gamma ray multiplicity spectrum were taken on-line with an IBM360 computer, using the extensive software developed by our collaborators from the University of Maryland.

As explained above we were interested in the neutrons emitted at $90^{\circ}$ with respect to the beam direction. Three long plastic scintillators of $24^{\prime \prime} \times 6^{\prime \prime} \times 1 \frac{1}{2}$ " in dimension were mounted in an arch over the target at a distance of $20^{\prime \prime}$ at the center, as shown in Figure 1. The thickness of $1 \frac{1,2}{2}$ was chosen as a compromise for a good TOF resolution, a good efficiency for the neutron detection, and a reasonable rejection of the gamma ray background. This thickness corresponds roughly to $50 \%$ efficiency for 2-MeV neutrons.

The experiment was intended to measure both small and large angle correlations, and a severe difficulty was encountered in bringing the scintillators close together and still have good light collection at earh end of the counter. We fabricated crossed adiabatic light guides out of interlacing lucite prisms in eight layers, achieving minimum angles of $16^{\circ}$ for the neutron-neutron correlation study. Using two fast $5^{\prime \prime}$ tubes, XP-2041, for both ends of the detectors we were able to obtain a position resolution of $3^{\prime \prime}$ and TOF resolution of $800 \mathrm{psec}$ using the timing provided by the cyclotron RF signal, which corresponded to $50 \mathrm{keV}$ energy resolution at the enutron energy of $1 \mathrm{MeV}$ and $1 \mathrm{MeV}$ resolution at $10 \mathrm{MeV}$. The discriminator threshold for each phototube was set at $300 \mathrm{keV}$ equivalent electron energy. 
The essential part of the electronics is shown in Figure 2, in which most of the usual components such as amplifiers and discriminators are eliminated for clarity, and only a portion of the repetitious features is shown. For convenience the coincidences between two phototubes on a single counter are termed the "singles" events, and coincidences between two singles events are termed the "coincidence" events. In this sense a coincidence event is a coincidence among four of the appropriate phototubes. Considering the rather high singles rate, the start pulse for each time-to-amplitude converter (TAC) was gated by the coincidence event signal.

Originally the gamma ray multiplicity counters were designed in order to provide flexible cuts of the correlation based on the gamma ray multiplicity, which is related to the angular momenta of the entry state into the yrast cascades. However, the role of multiplicity counters was reduced during the last run to an effective trigger for the heavily damped reaction, because of the low beam current. In the design of the multiplicity counters, priorities were given to the effective elimination of neutrons by time-of-flight, and ability to detect at least one gamma ray for each fusion-like reaction. The first requirement was best achieved by using fast plastic scintillators, and the second by using as many counters as possible at close range. We used 16 of Pilot $B$ scintillators, $2 " \times 2 "$ in dimension, mounted on 56AVP's at 8 "from the target. The discrimination level was set approximately at $300 \mathrm{keV}$, and all sixteen counters were timed within 1 nanosecond. The analog signal from a majority logic unit receiving the sixteen counter signals 


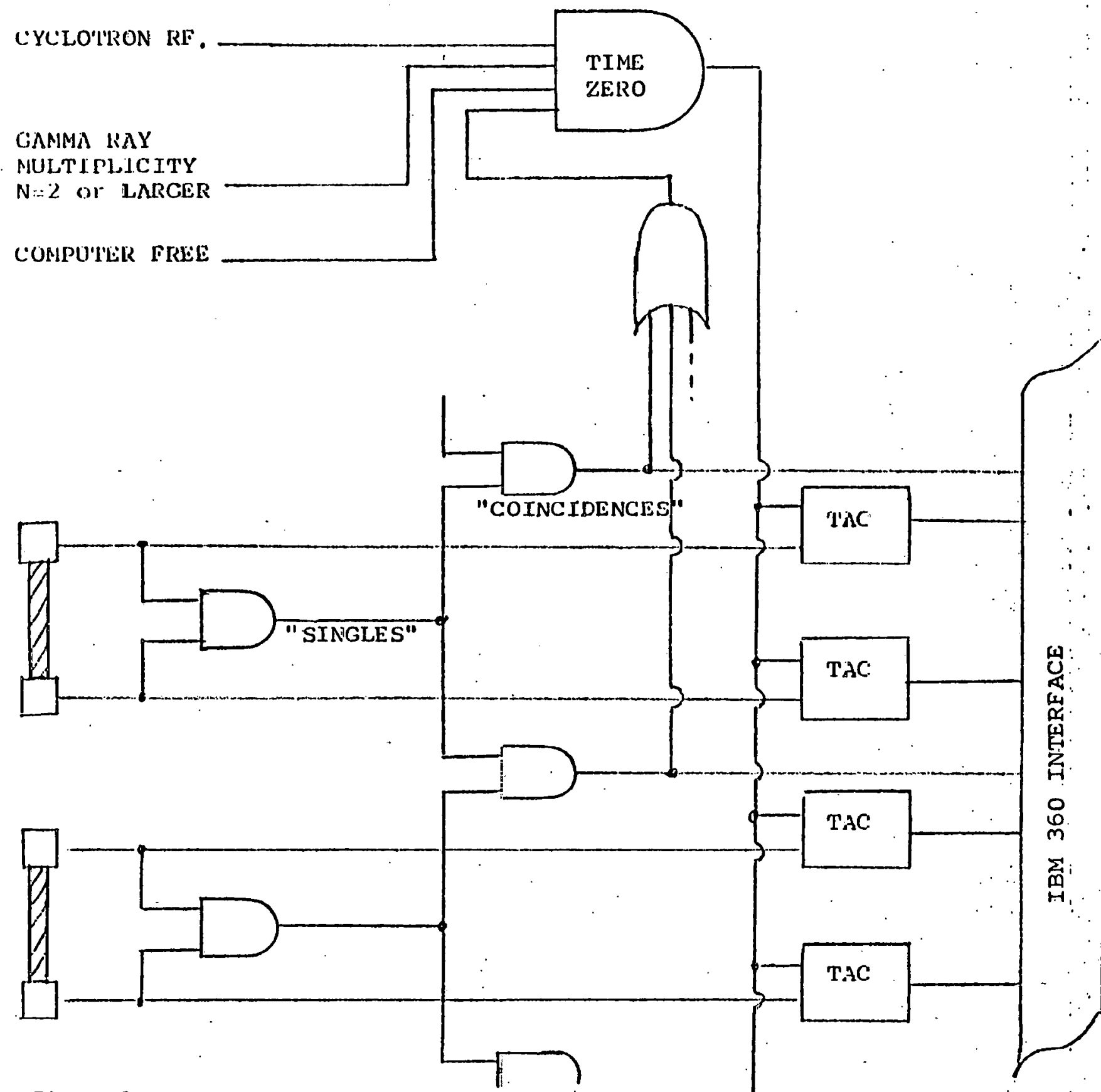

Figure 2 . only a part of the repetitious arrangement is shown. 
was recorded in the computer, and the logic signal for the multiplicity of 2 or more was included in the master gate.

The on-line IBM360 produced an event tape, and additionally kept several real-time histograms. The singles time-of-flight for each phototube, and the mean time-of-flight for each paddle, were available. Several two-dimensional histograms were available, which allowed an on-line check of our coincidence requirements.

The target was a $92 \%$ enriched, self-supporting metallic ${ }^{158} \mathrm{Gd}$, which was $2 \mathrm{mg} / \mathrm{cm}^{2}$ thick and mounted on a hole of $3 / 4$ " in diameter.

RESULTS

The previous work on the fusion-like reaction with ${ }^{158} \mathrm{Gd}+{ }^{12} \mathrm{C}$ indicated that the contribution from the pre-equilibrium components became significant at the excitation energy of $100 \mathrm{MeV}$ in the center of mass system of the product nuclei. ${ }^{2}$ We chose to develop a $120-\mathrm{MeV}$ beam of ${ }^{12} \mathrm{C}$ at the isochronous cyclotron of the University of Maryland with the help of Dr. D. A. Goldberg. This was the first heavy-ion beam . ever developed at the facility, and we were not able to exceed the beam current of 20 particle-pecoampere before the facility was permanently shut down in June, 1980. Another major obstacle was the poor vacuum in their beam transport system which was adequate perhaps for light particle beams but played havoc with the heavy-ion beam.

Implication of these difficulties is that we could not include the discrete gamma-ray spectra in the correlation, and that the correlation among the energies of two neutrons, $E_{1}$ and $E_{2}$, and the angles between 
them, $\theta=\theta_{1}-\theta_{2}$, was rather limited. We were not able to use the full resolution of our TOF. system nor the gamma ray multiplicity cuts during the experiments at the University of Maryland.

The response function of the overall TOF system for the detection of a coincidence event between two correlated neutrons is best obtained by constructing histograms based on uncorrelated neutrons but with the same energy and angle parameters $E_{1}, E_{2}$, and $\theta=\theta_{1}-\theta_{2}$. The pair of uncorrelated neutrons were picked by a Monte Carlo program from the same TOF data as used for the correlated neutrons. Therefore we constructed a second set of three-dimensional arrays for the uncorrelated events using the same three parameters in order to determine the response of the system and normalize our coincidence data.

The above method of analys is is best illustrated in the Tables 1 to 3 . Here we try to analyze the correlations between two momenta $P_{1}$ and $P_{2}$ of the coincident neutrons after summing all angles. Table 1 shows the coincidence events. The resolution of the momentum space corresponds to the timing resolution of 1 nanosecond. Most of the coincidence events are shown to be in the upper left corner corresponding to the statistical emission. The ridge due to residual activities not related to the time structure of the beam is seen to run diagonally across the table, with a width roughly corresponding to the dimension of the TOF system divided by the speed of light. The uncorrelated events obtained by the Monte Carlo type calculation is in Table 2. Table 3 shows the coincidence events normalized by the Monte Carlo type calculation. We notice that, within the statistics of the present experiment, 
Table 1. Sample Two-dimensional Spectra for Correlated Neutrons Gated by Gamma Ray Multiplicity $M \geq 2$

\begin{tabular}{|c|c|c|c|c|c|c|c|c|c|c|c|c|c|c|c|c|c|}
\hline $\begin{array}{c}\mathrm{P}_{1} \\
\mathrm{MeV} / \mathrm{C} \\
\mathrm{PeV} / \mathrm{C}\end{array}$ & 40 & 50 & 60 & 70 & 80 & 90 & 100 & 110 & 120 & 130 & 140 & 150 & 160 & 170 & 180 & 190 & 200 \\
\hline $\begin{array}{l}40 \\
50 \\
60\end{array}$ & $\begin{array}{r}855 \\
1538 \\
1547\end{array}$ & $\begin{array}{l}1535 \\
3263 \\
3191\end{array}$ & $\begin{array}{l}1392 \\
3304 \\
3144\end{array}$ & $\begin{array}{r}933 \\
2201 \\
1905\end{array}$ & $\begin{array}{r}548 \\
1199 \\
1071\end{array}$ & $\begin{array}{l}276 \\
643 \\
559\end{array}$ & $\begin{array}{l}148 \\
260 \\
279\end{array}$ & $\begin{array}{r}76 \\
149 \\
134\end{array}$ & $\begin{array}{l}37 \\
82 \\
85\end{array}$ & $\begin{array}{l}36 \\
64 \\
63\end{array}$ & $\begin{array}{l}29 \\
66 \\
56\end{array}$ & $\begin{array}{l}43 \\
69 \\
77\end{array}$ & $\begin{array}{l}47 \\
82 \\
62\end{array}$ & $\begin{array}{l}52 \\
97 \\
91\end{array}$ & $\begin{array}{r}55 \\
123 \\
92\end{array}$ & $\begin{array}{r}62 \\
134 \\
107\end{array}$ & $\begin{array}{r}60 \\
143 \\
138\end{array}$ \\
\hline $\begin{array}{l}70 \\
80 \\
90\end{array}$ & $\begin{array}{l}942 \\
554 \\
260\end{array}$ & $\begin{array}{r}2055 \\
1113 \\
529\end{array}$ & $\begin{array}{r}2026 \\
1112 \\
519\end{array}$ & $\begin{array}{r}1321 \\
706 \\
365\end{array}$ & $\begin{array}{l}712 \\
407 \\
214\end{array}$ & $\begin{array}{l}349 \\
217 \\
125\end{array}$ & $\begin{array}{r}159 \\
76 \\
49\end{array}$ & $\begin{array}{l}71 \\
36 \\
38\end{array}$ & $\begin{array}{l}52 \\
31 \\
18\end{array}$ & $\begin{array}{r}40 \\
19 \\
6\end{array}$ & $\begin{array}{r}50 \\
23 \\
\cdot \quad 7\end{array}$ & $\begin{array}{l}46 \\
22 \\
13\end{array}$ & $\begin{array}{l}43 \\
20 \\
15\end{array}$ & $\begin{array}{r}48 \\
22 \\
7\end{array}$ & $\begin{array}{l}76 \\
28 \\
12\end{array}$ & $\begin{array}{l}60 \\
28 \\
13\end{array}$ & $\begin{array}{l}75 \\
48 \\
14\end{array}$ \\
\hline $\begin{array}{l}100 \\
110 \\
120\end{array}$ & $\begin{array}{r}117 \\
57 \\
47\end{array}$ & $\begin{array}{r}225 \\
151 \\
92\end{array}$ & $\begin{array}{r}229 \\
129 \\
72\end{array}$ & $\begin{array}{r}160 \\
84 \\
64\end{array}$ & $\begin{array}{r}104 \\
35 \\
30\end{array}$ & $\begin{array}{l}62 \\
26 \\
17\end{array}$ & $\begin{array}{l}49 \\
26 \\
13\end{array}$ & $\begin{array}{l}35 \\
16 \\
13\end{array}$ & $\begin{array}{l}13 \\
22 \\
15\end{array}$ & $\begin{array}{l}11 \\
12 \\
14\end{array}$ & $\begin{array}{r}7 \\
10 \\
12\end{array}$ & $\begin{array}{l}8 \\
5 \\
9\end{array}$ & $\begin{array}{r}2 \\
6 \\
10\end{array}$ & $\begin{array}{l}9 \\
5 \\
3\end{array}$ & $\begin{array}{r}13 \\
5 \\
5\end{array}$ & $\begin{array}{l}8 \\
7 \\
1\end{array}$ & $\begin{array}{l}9 \\
5 \\
5\end{array}$ \\
\hline $\begin{array}{l}130 \\
140 \\
150\end{array}$ & $\begin{array}{l}40 \\
34 \\
36\end{array}$ & $\begin{array}{l}63 \\
65 \\
61\end{array}$ & $\begin{array}{l}50 \\
63 \\
58\end{array}$ & $\begin{array}{l}33 \\
33 \\
41\end{array}$ & $\begin{array}{l}20 \\
20 \\
20\end{array}$ & $\begin{array}{l}11 \\
11 \\
12\end{array}$ & $\begin{array}{r}10 \\
7 \\
8\end{array}$ & $\begin{array}{l}8 \\
6 \\
6\end{array}$ & $\begin{array}{l}7 \\
7 \\
9\end{array}$ & $\begin{array}{r}23 \\
16 \\
4\end{array}$ & $\begin{array}{l}15 \\
19 \\
14\end{array}$ & $\begin{array}{l}11 \\
12 \\
26\end{array}$ & $\begin{array}{r}4 \\
3 \\
10\end{array}$ & $\begin{array}{r}4 \\
4 \\
11\end{array}$ & $\begin{array}{l}4 \\
7 \\
4\end{array}$ & $\begin{array}{l}3 \\
4 \\
4\end{array}$ & $\begin{array}{l}3 \\
3 \\
2\end{array}$ \\
\hline $\begin{array}{l}160 \\
170 \\
180\end{array}$ & $\begin{array}{l}47 \\
48 \\
66\end{array}$ & $\begin{array}{r}83 \\
95 \\
108\end{array}$ & $\begin{array}{l}72 \\
85 \\
97\end{array}$ & $\begin{array}{l}36 \\
47 \\
58\end{array}$ & $\begin{array}{l}22 \\
29 \\
20\end{array}$ & $\begin{array}{l}12 \\
11 \\
14\end{array}$ & $\begin{array}{l}5 \\
7 \\
5\end{array}$ & $\begin{array}{l}6 \\
4 \\
4\end{array}$ & $\begin{array}{r}7 \\
10 \\
2\end{array}$ & $\begin{array}{l}6 \\
7 \\
4\end{array}$ & $\begin{array}{r}10 \\
3 \\
6\end{array}$ & $\begin{array}{r}20 \\
11 \\
4\end{array}$ & $\begin{array}{l}24 \\
27 \\
14\end{array}$ & $\begin{array}{l}17 \\
31 \\
28\end{array}$ & $\begin{array}{l}17 \\
26 \\
25\end{array}$ & $\begin{array}{r}5 \\
9 \\
22\end{array}$ & $\begin{array}{r}9 \\
5 \\
17\end{array}$ \\
\hline $\begin{array}{l}190 \\
200\end{array}$ & $\begin{array}{l}67 \\
74\end{array}$ & $\begin{array}{l}118 \\
120\end{array}$ & $\begin{array}{r}95 \\
118\end{array}$ & $\begin{array}{l}66 \\
65\end{array}$ & $\begin{array}{l}39 \\
52\end{array}$ & $\begin{array}{l}10 \\
15\end{array}$ & $\begin{array}{l}5 \\
4\end{array}$ & $\begin{array}{l}5 \\
5\end{array}$ & $\begin{array}{l}3 \\
4\end{array}$ & $\begin{array}{l}4 \\
4\end{array}$ & $\begin{array}{l}4 \\
1\end{array}$ & $\begin{array}{l}5 \\
5\end{array}$ & $\begin{array}{r}12 \\
6\end{array}$ & $\begin{array}{l}19 \\
11\end{array}$ & $\begin{array}{l}35 \\
20\end{array}$ & $\begin{array}{l}32 \\
29\end{array}$ & $\begin{array}{l}27 \\
40\end{array}$ \\
\hline
\end{tabular}


Table 2. Sample Monte Carlo Calculation of the Two-dimensional Spectra for Uncorrelated Neutrons

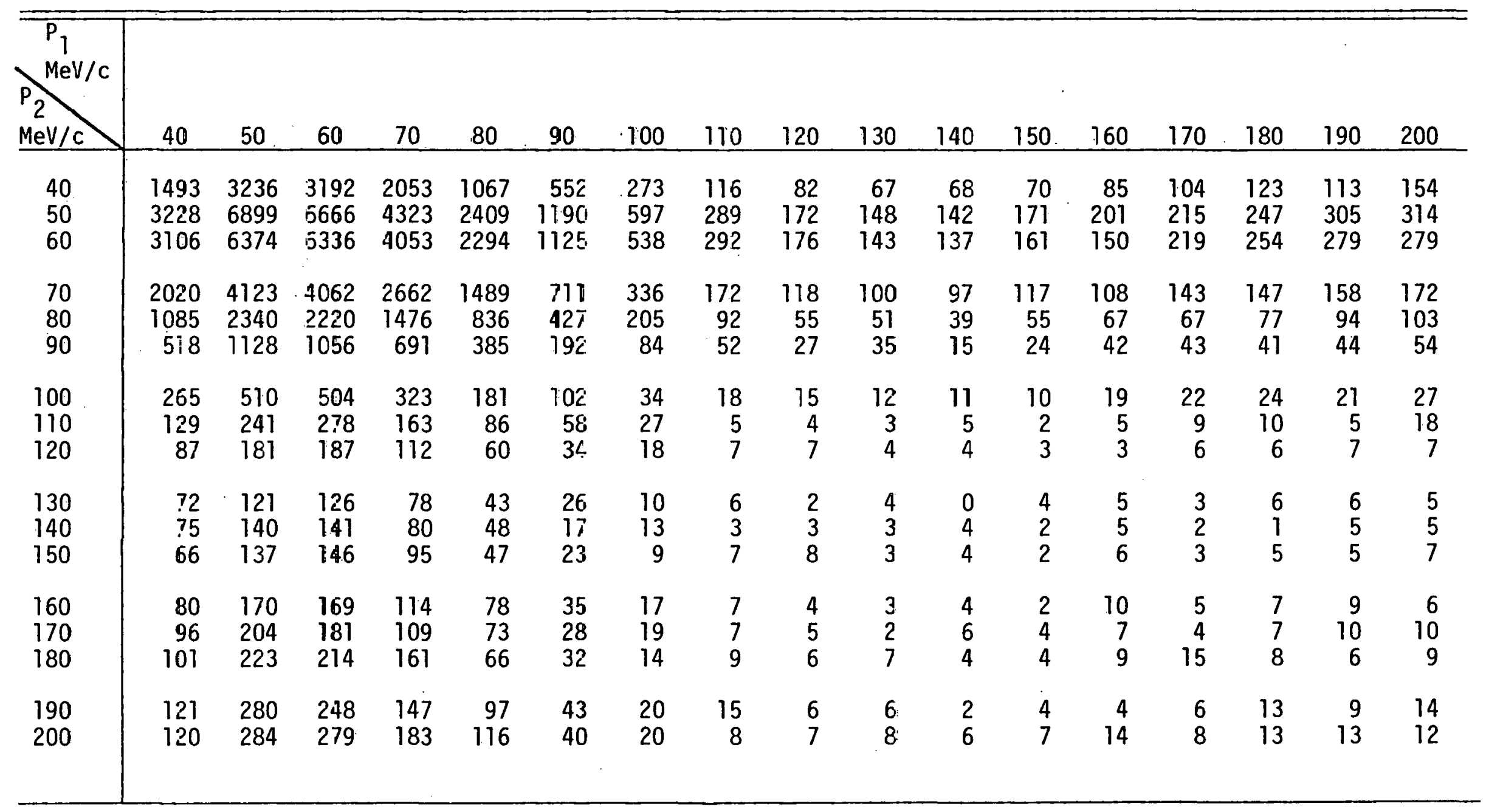


Table 3. Normalization of the Correlated Spectra (in Table 1) by the Monte Carlo Uncorrelated Spectra (in Table 2)

\begin{tabular}{|c|c|c|c|c|c|c|c|c|c|c|c|c|c|c|c|c|c|}
\hline $\begin{array}{c}\mathrm{P}_{1} \\
\mathrm{M} \in \mathrm{V} \\
\mathrm{P}_{2} \\
\mathrm{MeV} / \mathrm{C}\end{array}$ & 40 & 50 & 60 & 70 & 80 & 90 & 100 & 110 & 120 & 130 & 140 & 150 & 160 & 170 & 180 & 190 & 200 \\
\hline $\begin{array}{l}40 \\
50 \\
60\end{array}$ & $\begin{array}{r}114 \\
95 \\
99\end{array}$ & $\begin{array}{r}94 \\
94 \\
100\end{array}$ & $\begin{array}{l}87 \\
99 \\
99\end{array}$ & $\begin{array}{r}90 \\
101 \\
96\end{array}$ & $\begin{array}{r}102 \\
99 \\
93\end{array}$ & $\begin{array}{r}100 \\
108 \\
99\end{array}$ & $\begin{array}{r}108 \\
87 \\
103\end{array}$ & $\begin{array}{r}131 \\
103 \\
91\end{array}$ & $\begin{array}{l}90 \\
95 \\
96\end{array}$ & $\begin{array}{r}107 \\
86 \\
88\end{array}$ & $\begin{array}{l}85 \\
92 \\
81\end{array}$ & $\begin{array}{r}122 \\
80 \\
95\end{array}$ & $\begin{array}{r}110 \\
82 \\
82\end{array}$ & $\begin{array}{r}100 \\
90 \\
83\end{array}$ & $\begin{array}{l}89 \\
99 \\
72\end{array}$ & $\begin{array}{r}109 \\
87 \\
76\end{array}$ & $\begin{array}{l}77 \\
91 \\
98\end{array}$ \\
\hline $\begin{array}{l}70 \\
80 \\
90\end{array}$ & $\begin{array}{r}93 \\
102 \\
100\end{array}$ & $\begin{array}{l}99 \\
95 \\
93\end{array}$ & $\begin{array}{r}99 \\
100 \\
98\end{array}$ & $\begin{array}{r}99 \\
95 \\
105\end{array}$ & $\begin{array}{r}95 \\
97 \\
111\end{array}$ & $\begin{array}{r}98 \\
101 \\
130\end{array}$ & $\begin{array}{r}94 \\
74 \\
116\end{array}$ & $\begin{array}{r}82 \\
78 \\
146\end{array}$ & $\begin{array}{r}88 \\
112 \\
133\end{array}$ & $\begin{array}{l}80 \\
74 \\
34\end{array}$ & $\begin{array}{r}103 \\
117 \\
93\end{array}$ & $\begin{array}{r}78 \\
80 \\
108\end{array}$ & $\begin{array}{l}79 \\
59 \\
71\end{array}$ & $\begin{array}{l}67 \\
65 \\
32\end{array}$ & $\begin{array}{r}103 \\
72 \\
58\end{array}$ & $\begin{array}{l}75 \\
59 \\
59\end{array}$ & $\begin{array}{l}87 \\
93 \\
51\end{array}$ \\
\hline $\begin{array}{l}100 \\
110 \\
120\end{array}$ & $\begin{array}{r}88 \\
88 \\
108\end{array}$ & $\begin{array}{r}88 \\
125 \\
101\end{array}$ & $\begin{array}{l}90 \\
92 \\
77\end{array}$ & $\begin{array}{r}99 \\
103 \\
114\end{array}$ & $\begin{array}{r}114 \\
81 \\
100\end{array}$ & $\begin{array}{r}121 \\
89 \\
100\end{array}$ & $\begin{array}{l}288 \\
192 \\
144\end{array}$ & $\begin{array}{l}388 \\
640 \\
371\end{array}$ & $\begin{array}{r}173 \\
1100 \\
428\end{array}$ & $\begin{array}{l}183 \\
800 \\
700\end{array}$ & $\begin{array}{l}127 \\
400 \\
600\end{array}$ & $\begin{array}{r}160 \\
500 \\
.600\end{array}$ & $\begin{array}{r}21 \\
240 \\
666\end{array}$ & $\begin{array}{r}81 \\
111 \\
100\end{array}$ & $\begin{array}{l}108 \\
100 \\
166\end{array}$ & $\begin{array}{r}76 \\
280 \\
28\end{array}$ & $\begin{array}{r}66 \\
55 \\
142\end{array}$ \\
\hline $\begin{array}{l}130 \\
140 \\
150\end{array}$ & $\begin{array}{r}111 \\
90 \\
109\end{array}$ & $\begin{array}{r}104 \\
92 \\
89\end{array}$ & $\begin{array}{l}79 \\
89 \\
79\end{array}$ & $\begin{array}{l}84 \\
82 \\
86\end{array}$ & $\begin{array}{l}93 \\
83 \\
85\end{array}$ & $\begin{array}{r}84 \\
129 \\
104\end{array}$ & $\begin{array}{l}200 \\
107 \\
177\end{array}$ & $\begin{array}{l}266 \\
400 \\
171\end{array}$ & $\begin{array}{l}700 \\
466 \\
225\end{array}$ & $\begin{array}{r}1150 \\
1066 \\
266\end{array}$ & $\begin{array}{r}\star \star \star \star \\
950 \\
700\end{array}$ & $\begin{array}{r}550 \\
1200 \\
2600\end{array}$ & $\begin{array}{l}160 \\
120 \\
333\end{array}$ & $\begin{array}{l}266 \\
400 \\
733\end{array}$ & $\begin{array}{r}133 \\
1400 \\
160\end{array}$ & $\begin{array}{l}100 \\
160 \\
160\end{array}$ & $\begin{array}{r}120 \\
120 \\
57\end{array}$ \\
\hline $\begin{array}{l}160 \\
170 \\
180\end{array}$ & $\begin{array}{l}117 \\
100 \\
130\end{array}$ & $\begin{array}{l}97 \\
93 \\
96\end{array}$ & $\begin{array}{l}85 \\
93 \\
90\end{array}$ & $\begin{array}{l}63 \\
86 \\
72\end{array}$ & $\begin{array}{l}56 \\
79 \\
60\end{array}$ & $\begin{array}{l}68 \\
78 \\
87\end{array}$ & $\begin{array}{l}58 \\
73 \\
71\end{array}$ & $\begin{array}{r}171 \\
114 \\
88\end{array}$ & $\begin{array}{r}350 \\
400 \\
66\end{array}$ & $\begin{array}{l}400 \\
700 \\
114\end{array}$ & $\begin{array}{l}500 \\
100 \\
300\end{array}$ & $\begin{array}{r}2000 \\
550 \\
200\end{array}$ & $\begin{array}{l}480 \\
771 \\
311\end{array}$ & $\begin{array}{r}680 \\
1550 \\
373\end{array}$ & $\begin{array}{l}485 \\
742 \\
625\end{array}$ & $\begin{array}{l}111 \\
180 \\
733\end{array}$ & $\begin{array}{l}300 \\
100 \\
377\end{array}$ \\
\hline $\begin{array}{l}190 \\
200\end{array}$ & $\begin{array}{l}110 \\
123\end{array}$ & $\begin{array}{l}84 \\
84\end{array}$ & $\begin{array}{l}76 \\
84\end{array}$ & $\begin{array}{l}89 \\
71\end{array}$ & $\begin{array}{l}80 \\
89 .\end{array}$ & $\begin{array}{l}46 \\
75\end{array}$ & $\begin{array}{l}50 \\
40\end{array}$ & $\begin{array}{r}66 \\
125\end{array}$ & $\begin{array}{l}100 \\
114\end{array}$ & $\begin{array}{l}133 \\
100\end{array}$ & $\begin{array}{r}400 \\
33\end{array}$ & $\begin{array}{l}250 \\
142\end{array}$ & $\begin{array}{r}600 \\
85\end{array}$ & $\begin{array}{l}633 \\
275\end{array}$ & $\begin{array}{l}538 \\
307\end{array}$ & $\begin{array}{l}7111 \\
446\end{array}$ & $\begin{array}{l}385 \\
666\end{array}$ \\
\hline
\end{tabular}


there is no correlation between two neutron momenta. The flatness gives us a confidence in the present method of the data handling. The diagonal ridge still remains, since the normalization is based on the uncorrelated events which will not represent the coincident background due to the residual activities as can be seen in Table 2. The ridge is overwhelmed by the high statistics in the statistical emission region in the upper left corner.

In order to make most out of the data which we were able to obtain under the present circumstances, the data are now being analyzed for the quantity

$$
R=\frac{\left\langle E_{1} E_{2}\right\rangle}{\left\langle E_{1}\right\rangle\left\langle E_{2}\right\rangle}
$$

where the averaging represents integration over the angles and energy of the neutrons. We are now computing the ratio $R$ based on our threedimensional spectra for both the correlated and uncorrelated events discussed above. We are also computing theoretical ratio based on the pre-equilibrium model code provided by Dr. George Chang of the University of Maryland. 5 The code essentially calculates the probability of the emission of the first neutron as a pre-equitibrium decay on the basis of the exiton model, and treats the subsequent decay as statistical. In this analysis also the compromised signal-to-noise ratio near the $E_{1}=E_{2}$ region due to gamma ray background poses serious problems. The current data indicate that the $E_{1}$ and $E_{2}$ correlation between two neutrons is consistent with the absence of pre-equilibrium neutrons for this reaction at this energy, but this conclusion should be 
qualified because of the preliminary nature of our runs at the University of Maryland Cyclotron. For any definite conclusion we should have a better heavy-ion beam, and perhaps at a higher energy such as at Oak Ridge National Laboratory where the full capability of the apparatus could be put to work. The experiment has been useful in testing out our time-of-flight arrangement and the gamma ray multiplicity counters, especially paving the way for the neutron and gamma ray correlation experiments following the $\pi^{-}$-capture to be staged at the TRIUMF accelerator next fall.

\section{REFERENCES :}

1. For a general review of the subject see: W. U. Schroder and J. R. Huizinga, Annual Reviews of Nuclear Sciences 27, 465 (1977).

2. D. G. Sarantites, et al., Phys. Rev. C18, 774 (1978); L. Westerberg, et al., Phys. Rev. C18, 796 (1978).

3. G. I. Kopylov and P. I. Podgoretskii, Soviet Journal of Nuclear Physics 15, 219 (1972).

4. W. Zajc et al., Bull. Am. Phys. Soc. $\underline{25}, 506$ (1980).

5. C. C. Chang, Code "PREEQ", private communication; also see, J. R. Wu and C. C. Chang, Phys. Rev. 16, 1812 (1977); Phys. Rev. 17, 1540 (1978). 
LIST OF PUBLICATIONS DURING THIS CONTRACT PERIOD:

R. L. Robinson, H. J. Kim, R. O. Sayer, W. T. Milner, R. B. Piercey, J. H. Hamilton, A. V. Ramayya, J. C. Wells, Jr., A. J. Caffrey, "High-spin levels and band structure in ${ }^{78} \mathrm{Kr}$," Phys. Rev. C2], $603(1980)$.

W. DeJarnette, T. Hallman, E. McIntyre, J. C. Walker, J. Carroll, A. Sagle, R. J. Semper, "Limits on Threshold Phenomena in Central Collisions of ${ }^{12} \mathrm{C}+\mathrm{Pb}$ from $1-2 \mathrm{GeV} / \mathrm{n}$," Physics Letters $B$, accepted for publication.

\section{ABSTRACTS :}

E. McIntyre, Jr., T. Hallman, W. DeJarnette, J. C. Walker, L. Madansky,

R. Semper, E. Whipple and J. B. Carroll, "Wide Angle Pion Production in ${ }^{12} \mathrm{C}+{ }^{208} \mathrm{~Pb}$ Central Collisions at Beam Energies Ranging from 1.05 to $2.1 \mathrm{GeV} / \mathrm{n}, "$ Bull. Am. Phys. Soc. 24,835 (1979).

T. Hallman, W. DeJarnette, E. McIntyre, Jr., J. C. Walker, L. Madansky, R. Semper, A. L. Sagle, E. Whipple and J. B. Carroll, "Characteristics of Neutral Mesun Production from Coll1sions of Relaw. tivistic ${ }^{12} \mathrm{C}$ and ${ }^{40} \mathrm{Ar}$ Ions on Lead," Bull. Am. Phys. Soc. 24, 835 (1979).

J. C. Wells, Jr., R. W. Eastes, M. E. Barclay, R. L. Robinson, H. J. Kim, R. 0. Sayer, R. B. Piercey, J. H. Hamilton, A. V. Ramayya, C. F. Maguire, A. J. Caffrey, "High-Spin States in ${ }^{76} \mathrm{Br}, " \mathrm{Bu} 11$. Am. Phys. Soc. 25, 578 (1980). 
D. C. Lu, L. Delker, G. Dugan, C. S. Wu, A. J. Caffrey, Y. T. Cheng, and Y. K. Lee, "New Precision Pionic Mass Measurement and MuNeutrino Mass Value from Pionic Atoms," Submitted to the 7th" International Conference on Atomic Physics, M.I.T., August 4-8, 1980. 